\title{
Four-point correlator constraints on electromagnetic chiral parameters and resonance effective lagrangians
}

\author{
Balasubramanian Ananthanarayan \\ Centre for High Energy Physics, Indian Institute of Science \\ Bangalore, 560 012, India \\ E-mail: anant@cts.iisc.ernet.in
}

\section{Bachir Moussallam}

Groupe de Physique Théorique, IPN

Université Paris-Sud, F-91406 Orsay Cédex, France

E-mail: moussall@ipno.in2p3.fr

ABSTRACT: We pursue the analysis of a set of generalized DGMLY sum rules for the electromagnetic chiral parameters at order $e^{2} p^{2}$ and discuss implications for effective lagrangians with resonances. We exploit a formalism in which charge spurions are introduced and treated as sources. We show that no inconsistency arises from anomalies up to quadratic order in the spurions. We focus on the sum rules associated with QCD 4-point correlators which were not analyzed in detail before. Convergence properties of the sum rules are deduced from a general analysis of the form of the counterterms in the presence of electromagnetic spurions. Following the approach in which vector and axial-vector resonances are described with antisymmetric tensor fields and have a chiral order, we show that the convergence constraints are violated at chiral order four and can be satisfied by introducing a set of terms of order six. The relevant couplings get completely and uniquely determined from a set of generalized Weinberg sum-rule relations. An update on the corrections to Dashen's low-energy theorem is given.

Keywords: Electromagnetic Processes and Properties, Sum Rules, Phenomenological Models, Chiral Lagrangians. 


\section{Contents}

1. Motivation 2

2. Chiral calculation of the basic correlators 3

2.1 Definition of the basic correlation functions

2.2 Correlators at order $e^{2} p^{2}$

2.3 Calculation of the one-loop photon contributions

3. Correlators from the minimal resonance lagrangian 11

$3.1 O\left(p^{4}\right)$ resonance lagrangian 11

3.2 Contributions proportional to $F_{A}^{2}$ and $F_{V}^{2}$

$3.3 G_{V} F_{V}$ contributions 14

$3.4 G_{V}^{2}$ and $G_{V}^{2} F_{V}^{2}$ contributions 16

3.5 Contributions proportional to $c_{d}, \tilde{c}_{d}$

3.6 Comparison with the results of Baur and Urech 17

4. Short distance constraints 18

4.1 QCD+QED counterterms 18

4.2 Summary of the $\log \Lambda^{2}$ dependence 20

5. Beyond the $O\left(p^{4}\right)$ resonance lagrangian 21

5.1 Survey of $O\left(p^{6}\right)$ terms 21

$5.2 \mathcal{L}_{a_{1} \rho \pi}$ constraints from $\langle V A P\rangle$

5.3 Linear contributions from $\mathcal{L}_{a_{1} \rho \pi}$

5.4 Quadratic contributions from $\mathcal{L}_{a_{1} \rho \pi}$ and consistency conditions for $\Pi_{1}, \Pi_{2}$, $\Pi_{8}$

5.5 Contributions from $\mathcal{L}_{a_{0} f_{1} \pi}$ and the consistency condition for $\Pi_{3}$

5.6 Contributions from $\mathcal{L}_{\omega \rho \pi}$ in the vector vs. tensor formalism and $\Pi_{4}$

6. Numerical results 30

6.1 Results for $K_{1}, \ldots, K_{6}$

6.2 An update on the corrections to Dashen's theorem 31

6.3 Uncertainties 32

7. Conclusions 33

A. Vertices 33

A.1 Vertices with pions 34

A.2 Resonance vertices with $v_{\mu}$ sources

A.3 Vertices proportional to $F_{A}$

A.4 Vertices proportional to $F_{V}$

A.5 Vertices proportional to $G_{V}$ 


\section{Motivation}

Virtual photons play an important role in low energy QCD for isospin breaking phenomena and must also be taken into account in connection with precision experiments. The formalism for extending the framework of the standard ChPT [1, 2, 3] to include virtual photons at one-loop was developed, some time ago, by Urech 酒. Several extensions were later performed to accommodate leptons [5], to the weak non-leptonic sector [6], to the anomalous sector [7] as well as to the baryon sector [8]. The problem of the determination of the new set of low-energy coupling constants (LEC's) which appear in Urech's lagrangian is of obvious practical importance in relation with radiative correction calculations. Many such calculations have been performed recently: low energy $\pi \pi$ or $\pi K$ scattering [- $[-10]$ pionic as well as pi-kaonic atoms [16, 17, 18], the set of semi-leptonic decays of the Kaon [19, 20, processes from the anomalous sector [21, 7], the problem of computing the hadronic contribution of the muon $g-2$ from $\tau$ decay data [22], the theoretical prediction of the CP-violation ratio $\epsilon^{\prime} / \epsilon$ for the Kaon [23, 24, 25] to mention just a few illustrative examples. Another important application of the electromagnetic low-energy couplings (LEC) is to the question of the chiral corrections to Dashen's low-energy theorem [26] and the determination of the light quark masses (e.g. [27]). Interesting questions were raised recently concerning the proper definition of the quark masses in the presence of QED [28.

The problem of the determination of the EM LEC's was addressed in several papers some time ago [29, 30, 31] but the results remain incomplete and sometimes contradictory. In this paper, we continue the discussion started in ref. [31] (hereafter referred to as (I)). In (I) it was shown that the electromagnetic LEC's obey integral sum rule representations which generalize the classic DGMLY sum rule [32] for the $\pi^{+}-\pi^{0}$ mass difference. The integral representations have the form of convolutions involving a pure QCD $n$-point Green's function with $n=2,3$ and 4 together with the free photon propagator. These representations serve a number of purposes. For instance, they can be used to recover the chiral scale dependence of the LEC's, to determine how these depend on the $\xi$ gauge parameter and, also, by studying the convergence properties, to determine which LEC's are affected by short distance ambiguities. Finally, these representations can be used as a method to provide approximate determinations of these couplings.

This was exploited in (I) to discuss the set of couplings which are associated with 2- and 3 -point Green's functions. The method consists in constructing rational approximants to the Green's functions, which is in the spirit of the large $N_{c}$ expansion [33]. The parameters of the rational functions are constrained by the chiral Ward identities, by physical input on resonance masses and couplings and by short-distance matching conditions. In the present paper, we address the more complicated case of the 4-point functions. The way of insuring that the Ward identities are satisfied is to start from a chiral lagrangian including 
resonances. Such a lagrangian was constructed in ref. [34 which uses the antisymmetric tensor formalism [2] to describe the vector and axial-vector resonances. This formalism is particularly convenient for discussing the ordinary LEC's because one can assign a chiral order to each resonance field such that all the terms which are of order four, which include one multiplet of vector, axial-vector, scalar and pseudo-scalar resonances were listed in ref. 34]. It was shown in a subsequent paper [35] that if one imposes a number of short distance matching conditions, the determination of the $O\left(p^{4}\right)$ LEC's becomes independent of the specific representation of the resonances in the chiral lagrangian.

The lagrangian of ref. [34] was employed by Baur and Urech [29] (BU) to discuss the electromagnetic LEC's. For this application, however, one realizes by using the integral representation that the Green's functions are needed under kinematical conditions which are different, in general, from those considered in [35] such that the lagrangian of ref. [34] is no longer sufficient to ensure the proper short distance constraints. This deficiency manifests itself in the fact that the results of BU for the electromagnetic LEC's fail to satisfy the correct scale dependence, which is an effect of leading order in $N_{c}$. In this paper, we will show that a simple extension of the lagrangian [34] to include a set of terms which are of chiral order six allows one to satisfy the short distance constraints in the chiral limit which are necessary for a consistent determination of the electromagnetic LEC's and which are associated with the 2- and 3-point functions considered in [31] as well as the 4-point functions introduced in this paper. Finally, we will present estimates for the six LEC's which are associated with these 4-point functions.

\section{Chiral calculation of the basic correlators}

\subsection{Definition of the basic correlation functions}

In the presence of a dynamical photon field $F_{\mu}$ it is convenient to extend the source part of the QCD lagrangian to include two spurion fields $q_{V}, q_{A}$ 顿

$$
\mathcal{L}_{\mathrm{QCD}}^{\text {sources }}=\bar{\psi}_{L} \gamma^{\mu}\left[l_{\mu}+q_{L} F_{\mu}\right] \psi_{L}+\bar{\psi}_{R} \gamma^{\mu}\left[r_{\mu}+q_{R} F_{\mu}\right] \psi_{R}-\bar{\psi}\left(s-i \gamma^{5} p\right) \psi
$$

with

$$
l_{\mu}=v_{\mu}-a_{\mu}, \quad q_{L}=q_{V}-q_{A}, \quad r_{\mu}=v_{\mu}+a_{\mu}, \quad q_{R}=q_{V}+q_{A} .
$$

The number of light flavours is assumed to be $N_{F}^{0}=3$, and the sources in eq. (2.2) are traceless $3 \times 3$ matrices. Originally [ [ 4 , the spurions $q_{V}, q_{A}$ were introduced only for the purpose of classifying the independent terms in the chiral lagrangian and afterwards one would set $q_{A}=0, q_{V}=Q$. We will exploit here more general applications by considering the spurions on the same footing as the ordinary external sources. We note that renormalization will generate terms which are non-linear in the sources. Those which are quadratic in the spurion sources are of particular interest for our purposes and will be discussed in detail in section 1 . It will also be argued that no difficulty arises in the definition of the generating functional in the presence of the $q_{V}, q_{A}$ spurions up to quadratic order in the spurions. We now introduce a set of five correlation functions defined by taking two functional derivatives 
with respect to $v_{\mu}$ or $a_{\mu}$ and two derivatives with respect to the charge spurions $q_{V}$ or $q_{A}$. The first one is defined as follows

$$
\text { 1. }\left\langle A_{\alpha}^{a} A_{\beta}^{b} Q_{A}^{c} Q_{A}^{d}\right\rangle=\int d^{4} x d^{4} y d^{4} z \exp (i k y) \frac{\delta^{4} W\left(v_{\mu}, a_{\mu}, q_{V}, q_{A}\right)}{\delta a_{\alpha}^{a}(x) \delta a_{\beta}^{b}(y) \delta q_{A}^{c}(z) \delta q_{A}^{d}(0)}
$$

where $W$ is the generating functional of connected Green's functions. The remaining four

$$
\begin{array}{ll}
\text { 2. }\left\langle A_{\alpha}^{a} A_{\beta}^{b} Q_{V}^{c} Q_{V}^{d}\right\rangle \\
\text { 3. }\left\langle V_{\alpha}^{a} V_{\beta}^{b} Q_{A}^{c} Q_{A}^{d}\right\rangle \\
\text { 4. }\left\langle V_{\alpha}^{a} V_{\beta}^{b} Q_{V}^{c} Q_{V}^{d}\right\rangle \\
\text { 5. }\left\langle A_{\alpha}^{a} V_{\beta}^{b} Q_{A}^{c} Q_{V}^{d}\right\rangle
\end{array}
$$

are defined in an exactly analogous way. These correlators involve two Lorentz structures $g_{\alpha \beta}$ and $k_{\alpha} k_{\beta}$. For our purposes, we can restrict ourselves to the Lorentz structure proportional to $g_{\alpha \beta}$ and we will consider the limit $k \rightarrow 0$. Before taking this limit, however, we must consider two possible kinds of singularities: a) $1 / k^{2}$ pion poles and b) $\log \left(k^{2}\right)$ cuts due to pion loops. The latter singularities drop out in the leading large $N_{c}$ approximation, which we will frequently invoke. A pion pole can appear only in one flavour structure of the fifth correlator $\left\langle A_{\alpha}^{a} V_{\beta}^{b} Q_{A}^{c} Q_{V}^{d}\right\rangle$. Apart from this particular case, we can simply set $k=0$, meaning that we restrict ourselves to constant vector and axial-vector sources. This leads to great simplification since all terms like $\partial_{\mu} v_{\nu}, \partial_{\mu} a_{\nu}$ etc... can be dropped in the chiral resonance lagrangian. Similarly, the $q_{V}$ and $q_{A}$ sources can be taken as constant for the present applications.

From the definition, eq. (2.3) and the explicit expression of the terms linear in the sources eq. (2.1) we can easily see that the correlators introduced above can be represented as a convolution of an ordinary connected QCD 4-point Green's function and the photon propagator. For example, one finds

$$
\left\langle A_{\alpha}^{a} A_{\beta}^{b} Q_{A}^{c} Q_{A}^{d}\right\rangle=\int d^{4} x d^{4} y d^{4} z \exp (i k y)\left\langle 0\left|T\left(A_{\alpha}^{a}(x) A_{\beta}^{b}(y) A_{\rho}^{c}(z) A_{\sigma}^{d}(0)\right)\right| 0\right\rangle D_{F}^{\rho \sigma}(z) .
$$

Because of the short distance singularities, the integral over $d^{4} z$ is expected to be divergent. The divergence is removed upon including the additional contributions stemming from the counterterms and one may use d-dimensional integration as a regularization method.

Let us now examine the flavour structure. The correlators depend on four flavour indices. At order $e^{2} p^{2}$ one can show by using the chiral lagrangian that there are only four independent tensor structures. It will prove convenient to use the following basis

$$
\begin{aligned}
& \mathbf{e}_{\mathbf{1}}=f^{t a c} f^{t b d}+f^{t a d} f^{t b c} \\
& \mathbf{e}_{\mathbf{2}}=\delta^{a c} \delta^{b d}+\delta^{a d} \delta^{b c} \\
& \mathbf{e}_{\mathbf{3}}=d^{t a c} d^{t b d}+d^{t a d} d^{t b c} \\
& \mathbf{e}_{\mathbf{4}}=f^{t a b} f^{t c d} .
\end{aligned}
$$


Other tensor structures that arise in practical calculations can be expressed in terms of this basis by utilizing $\mathrm{SU}(3)$ trace identities. The following relations are useful

$$
\begin{aligned}
d^{t a b} d^{t c d} & =\frac{1}{6} \mathbf{e}_{1}+\frac{1}{3} \mathbf{e}_{2}-\frac{1}{2} \mathbf{e}_{3} \\
\delta^{a b} \delta^{c d} & =\frac{1}{2} \mathbf{e}_{1}+\frac{3}{2} \mathbf{e}_{3} .
\end{aligned}
$$

We can now expand the correlators over the flavour basis. The first four correlators are expanded over $\mathbf{e}_{\mathbf{1}}, \mathbf{e}_{\mathbf{2}}, \mathbf{e}_{\mathbf{3}}$ because of the permutation symmetry $a \leftrightarrow b, c \leftrightarrow d$. For instance, one has

$$
\left\langle A_{\alpha}^{a} A_{\beta}^{b} Q_{A}^{c} Q_{A}^{d}\right\rangle=i g_{\alpha \beta} \sum_{j=1}^{3}\left\langle A A Q_{A} Q_{A}\right\rangle_{j} \mathbf{e}_{\mathbf{j}} .
$$

The expansion of the last correlator involves $\mathbf{e}_{\mathbf{4}}$ also,

$$
\left\langle A_{\alpha}^{a} V_{\beta}^{b} Q_{A}^{c} Q_{V}^{d}\right\rangle=i g_{\alpha \beta} \sum_{j=1}^{4}\left\langle A V Q_{A} Q_{V}\right\rangle_{j} \mathbf{e}_{\mathbf{j}} .
$$

This expansion apparently generates sixteen independent flavour components. As we will see below there are only ten components which appear at the chiral order $e^{2} p^{2}$ considered here.

\subsection{Correlators at order $e^{2} p^{2}$}

We start by computing the correlators defined above (2.3) (2.4) in the chiral expansion up to the order $e^{2} p^{2}$. Setting the sources $s$ and $p$ equal to zero, the leading order lagrangian collects the terms of order $e^{2}$ and $p^{2}$

$$
\begin{aligned}
& \mathcal{L}_{\text {chir }}^{(2)}=\mathcal{L}_{\text {chir }}^{p^{2}}+\mathcal{L}_{\text {chir }}^{e^{2}} \\
& \mathcal{L}_{\text {chir }}^{p^{2}}=\frac{F_{0}^{2}}{4}\left\langle D_{\mu} U D^{\mu} U^{\dagger}\right\rangle-\frac{1}{4} F_{\mu \nu} F^{\mu \nu}-\frac{1}{2 \xi}\left(\partial_{\mu} F^{\mu}\right)^{2}+\frac{1}{2} M_{\gamma}^{2} F_{\mu} F^{\mu} \\
& \mathcal{L}_{\text {chir }}^{e^{2}}=C\left\langle q_{L} U^{\dagger} q_{R} U\right\rangle,
\end{aligned}
$$

with

$$
D_{\mu} U=\partial_{\mu} U-i\left(r_{\mu}+q_{R} F_{\mu}\right) U+i U\left(l_{\mu}+q_{L} F_{\mu}\right) .
$$

The photon is endowed with a small mass (which may be counted as $O(p)$ ) in order to regulate infrared divergences which can appear in the chiral limit. We will also need the following part of the chiral lagrangian of order $e^{2} p^{2}$ [4]

$$
\begin{aligned}
\mathcal{L}_{\text {chir }}^{e^{2} p^{2}}= & \frac{1}{2} K_{1} F_{0}^{2}\left\langle D_{\mu} U D^{\mu} U^{\dagger}\right\rangle\left\langle q_{L} q_{L}+q_{R} q_{R}\right\rangle+ \\
& +K_{2} F_{0}^{2}\left\langle D_{\mu} U D^{\mu} U^{\dagger}\right\rangle\left\langle q_{L} U^{\dagger} q_{R} U\right\rangle+ \\
& +K_{3} F_{0}^{2}\left(\left\langle D_{\mu} U q_{L} U^{\dagger}\right\rangle^{2}+\left\langle D_{\mu} U^{\dagger} q_{R} U\right\rangle^{2}\right)+ \\
& +K_{4} F_{0}^{2}\left\langle D_{\mu} U q_{L} U^{\dagger}\right\rangle\left\langle D_{\mu} U^{\dagger} q_{R} U\right\rangle+ \\
& +K_{5} F_{0}^{2}\left\langle D_{\mu} U^{\dagger} D^{\mu} U q_{L} q_{L}+D_{\mu} U D^{\mu} U^{\dagger} q_{R} q_{R}\right\rangle+
\end{aligned}
$$




$$
\begin{aligned}
& +K_{6} F_{0}^{2}\left\langle D_{\mu} U^{\dagger} D^{\mu} U q_{L} U^{\dagger} q_{R} U+D_{\mu} U D^{\mu} U^{\dagger} q_{R} U q_{L} U^{\dagger}\right\rangle+ \\
& +K_{12} F_{0}^{2}\left\langle U D^{\mu} U^{\dagger}\left[\nabla_{\mu} q_{R}, q_{R}\right]+U^{\dagger} D^{\mu} U\left[\nabla_{\mu} q_{L}, q_{L}\right]\right\rangle+ \\
& +K_{13} F_{0}^{2}\left\langle\nabla_{\mu} q_{R} U \nabla^{\mu} q_{L} U^{\dagger}\right\rangle+ \\
& +K_{14} F_{0}^{2}\left\langle\nabla_{\mu} q_{L} \nabla^{\mu} q_{L}+\nabla_{\mu} q_{R} \nabla^{\mu} q_{R}\right\rangle
\end{aligned}
$$

where

$$
\nabla_{\mu} q_{L}=\partial_{\mu} q_{L}-i\left[l_{\mu}, q_{L}\right], \quad \nabla_{\mu} q_{R}=\partial_{\mu} q_{R}-i\left[l_{\mu}, q_{R}\right] .
$$

As usual, the correlators collect the contributions at one-loop generated from $\mathcal{L}_{\text {chir }}^{(2)}$ and the tree contributions from $\mathcal{L}_{\text {chir }}^{e^{2} p^{2}}$. Let us first quote the results, some details about how the calculation proceeds will be given later. For the components of $\left\langle A A Q_{A} Q_{A}\right\rangle$ one obtains

$$
\begin{aligned}
&\left\langle A A Q_{A} Q_{A}\right\rangle_{1}=F_{0}^{2}[ 2\left(K_{1}^{r}-K_{2}^{r}\right)+2\left(K_{5}^{r}-K_{6}^{r}\right)+4 K_{12}^{r}+K_{13}^{r}+2 K_{14}^{r}+ \\
&\left.+\chi_{1}\left(M_{\gamma}, \mu\right)+\frac{5}{4} Z(k, \mu)\right] \\
&\left\langle A A Q_{A} Q_{A}\right\rangle_{2}=F_{0}^{2}\left[-2\left(2 K_{3}^{r}+K_{4}^{r}\right)+\frac{4}{3}\left(K_{5}^{r}-K_{6}^{r}\right)+\frac{3}{2} Z(k, \mu)\right] \\
&\left\langle A A Q_{A} Q_{A}\right\rangle_{3}=F_{0}^{2}\left[6\left(K_{1}^{r}-K_{2}^{r}\right)+2\left(K_{5}^{r}-K_{6}^{r}\right)+\frac{9}{4} Z(k, \mu)\right]
\end{aligned}
$$

where $K_{i}^{r}$ stand for the renormalized scale dependent parameters. The function $\chi_{1}\left(M_{\gamma}, \mu\right)$ is one of $\chi_{i}, i=1,2,3,4$ which are the one-loop chiral contributions involving a virtual photon. We will describe the computation in detail in the next section. The contributions proportional to the function $Z(k, \mu)$ arise from purely pionic one-loop diagrams having one vertex from the term proportional to $C$ in eq. (2.10). This function reads

$$
Z(k, \mu)=\frac{2 C}{16 \pi^{2} F_{0}^{4}}\left(-\log \frac{-k^{2}}{\mu^{2}}+1\right) .
$$

While we include this contribution here for completeness we note that it is of subleading order in the large $N_{c}$ expansion. In the following, we will drop such contributions for consistency with the leading large $N_{c}$ approximation which we will make in the treatment of the resonances. We also note that the correlators must be independent of the chiral renormalization scale $\mu$. This constraint allows one to recover the scale dependence of $K_{i}^{r}(\mu)$ computed in refs. [四, 37], generalized to an arbitrary $\xi$ gauge. The components of the second correlator has the following expression

$$
\begin{aligned}
\left\langle A A Q_{V} Q_{V}\right\rangle_{1}=F_{0}^{2}[ & 2\left(K_{1}^{r}+K_{2}^{r}\right)+2\left(K_{5}^{r}+K_{6}^{r}\right)+4 K_{12}^{r}-K_{13}^{r}+2 K_{14}^{r}+ \\
& \left.+\chi_{2}\left(M_{\gamma}, \mu\right)-\frac{5}{4} Z(k, \mu)\right] \\
\left\langle A A Q_{V} Q_{V}\right\rangle_{2}=F_{0}^{2}\left[-2\left(2 K_{3}^{r}-K_{4}^{r}\right)+\frac{4}{3}\left(K_{5}^{r}+K_{6}^{r}\right)-\frac{3}{2} Z(k, \mu)\right] & {\left[6 Q_{V} Q_{V}\right\rangle_{3}=F_{0}^{2}\left[6\left(K_{1}^{r}+K_{2}^{r}\right)+2\left(K_{5}^{r}+K_{6}^{r}\right)-\frac{9}{4} Z(k, \mu)\right] . }
\end{aligned}
$$


For the next two correlators, only the first flavour structure appears at this chiral order

$$
\begin{aligned}
\left\langle V V Q_{A} Q_{A}\right\rangle_{1} & =F_{0}^{2}\left[-K_{13}^{r}+2 K_{14}^{r}+\chi_{3}\left(M_{\gamma}, \mu\right)\right] \\
\left\langle V V Q_{V} Q_{V}\right\rangle_{1} & =F_{0}^{2}\left[K_{13}^{r}+2 K_{14}^{r}\right] .
\end{aligned}
$$

The last correlator has two non-vanishing flavour structures. The structure proportional to $\mathbf{e}_{4}$ is the only one to receive a tree-level contribution from $\mathcal{L}_{\text {chir }}^{(2)}$,

$$
\begin{aligned}
\left\langle A V Q_{A} Q_{V}\right\rangle_{1} & =F_{0}^{2}\left[2 K_{12}^{r}+2 K_{14}^{r}+\chi_{4}\left(M_{\gamma}, \mu\right)\right] \\
\left\langle A V Q_{A} Q_{V}\right\rangle_{4} & =\frac{2 C}{k^{2}}+F_{0}^{2}\left[-K_{13}^{r}+2 K_{12}^{r}+\chi_{4}\left(M_{\gamma}, \mu\right)\right] .
\end{aligned}
$$

We now introduce a set of combinations of these correlator components which enjoy useful properties

$$
\begin{aligned}
& \Pi_{1}=\left\langle A A Q_{A} Q_{A}\right\rangle_{1}-2\left\langle A V Q_{A} Q_{V}\right\rangle_{1}+\left\langle V V Q_{A} Q_{A}\right\rangle_{1} \\
& \Pi_{2}=\left\langle A A Q_{V} Q_{V}\right\rangle_{1}-2\left\langle A V Q_{A} Q_{V}\right\rangle_{1}+\left\langle V V Q_{V} Q_{V}\right\rangle_{1} \\
& \Pi_{3}=\left\langle A A Q_{A} Q_{A}\right\rangle_{3} \\
& \Pi_{4}=\left\langle A A Q_{V} Q_{V}\right\rangle_{3} \\
& \Pi_{5}=\left\langle A A Q_{A} Q_{A}\right\rangle_{2}-\frac{2}{3}\left\langle A A Q_{A} Q_{A}\right\rangle_{3} \\
& \Pi_{6}=\left\langle A A Q_{V} Q_{V}\right\rangle_{2}-\frac{2}{3}\left\langle A A Q_{V} Q_{V}\right\rangle_{3} .
\end{aligned}
$$

These six combinations are in one to one correspondence with the parameters $K_{1}^{r}, \ldots, K_{6}^{r}$ which we intend to determine. One can show (see [31]) that they are independent of the gauge parameter $\xi$, and we will show below that they get no contributions from the QCD counterterms. This last fact implies that the photon loop integral must be converging. Convergence must be understood in the sense that integration is to be performed in $d$ dimensions and the limit $d \rightarrow 4$ is well defined.

In the leading large $N_{c}$ approximation one has

$$
\Pi_{5}=\Pi_{6}=0 \quad\left(\text { large } N_{c}\right) .
$$

This is not difficult to show. The graphs which are of leading order in $N_{c}$ are the planar ones which involve a single fermion loop [33. This implies that they involve a single trace over the flavour $\lambda$ matrices. Furthermore, we are interested in four-point QCD correlators which are symmetric under permutation of the Lorentz indices $\alpha, \beta$ as well as in the other two indices $\rho \sigma$ (see eq. (2.5)). They must then have the following structure

$$
\begin{aligned}
\left\langle A_{\alpha}^{a} A_{\beta}^{b} Q_{A}^{c} Q_{A}^{d}\right\rangle & =i g_{\alpha \beta}\left(E\left\langle\left\{\lambda^{a}, \lambda^{b}\right\}\left\{\lambda^{c}, \lambda^{d}\right\}\right\rangle+F\left\langle\lambda^{a} \lambda^{c} \lambda^{b} \lambda^{d}+\lambda^{a} \lambda^{d} \lambda^{b} \lambda^{c}\right\rangle\right) \\
& =i g_{\alpha \beta}\left(2(2 E-F) \mathbf{e}_{\mathbf{1}}+\frac{4}{3}(2 E+F)\left(\mathbf{e}_{\mathbf{2}}+\frac{3}{2} \mathbf{e}_{\mathbf{3}}\right)\right)
\end{aligned}
$$

making use of relations (2.7), where $E$ and $F$ are arbitrary, and the same holds for the propagator with $Q_{A} Q_{A}$ replaced by $Q_{V} Q_{V}$. This proves relations (2.20). From these, one can recover the large $N_{c}$ relations among the corresponding $K_{i}^{r}$ parameters

$$
K_{3}^{r}=-K_{1}^{r} \quad K_{4}^{r}=2 K_{2}^{r} \quad\left(\text { large } N_{c}\right)
$$


noted in refs. [㘬 and [30]. We will further consider the following three combinations

$$
\begin{aligned}
& \Pi_{7}=\left\langle V V Q_{V} Q_{V}\right\rangle_{1}-\left\langle V V Q_{A} Q_{A}\right\rangle_{1} \\
& \Pi_{8}=\left\langle A V Q_{A} Q_{V}\right\rangle_{1}-\left\langle V V Q_{V} Q_{V}\right\rangle_{1} \\
& \Pi_{9}=\lim _{k^{2} \rightarrow 0} k^{2}\left\langle A V Q_{A} Q_{V}\right\rangle_{4} .
\end{aligned}
$$

From these combinations we will recover established results concerning the couplings $C$, $K_{12}^{r}, K_{13}^{r} . \Pi_{7}$ and $\Pi_{8}$ depend on the gauge. Convergence of the photon loop integral can be established for $\Pi_{7}$ and $\Pi_{9}$ while for $\Pi_{8}$ it holds in the particular gauge $\xi=0$ and in a limited sense, as we will discuss in more detail. This limitation affects the determination of $K_{12}^{r}$. We will not attempt to estimate the coupling $K_{14}^{r}$ which is a pure source term.

One further remark is in order. One usually expects the coupling constants in ChPT to be associated with QCD correlators which are order parameters, except for the pure source terms. Looking at eqs. (2.19) this, at first sight, seems sometimes not to be the case here. For instance, $\Pi_{3}$ is associated with the correlator $\left\langle A_{\alpha}^{a} A_{\beta}^{b} A_{\mu}^{c} A_{\nu}^{d}\right\rangle$ which does not vanish in perturbation theory. However, this is simply due to the fact that the components $\left\langle A V Q_{A} Q_{V}\right\rangle_{3}$ and $\left\langle V V Q_{V} Q_{V}\right\rangle_{3}$ vanish at chiral order $e^{2} p^{2}$, so one could equally well express $\Pi_{3}$ in terms of a correlator which does behave as an order parameter.

\subsection{Calculation of the one-loop photon contributions}

In this section, we will compute contributions to the generating functional $W\left(v_{\mu}, a_{\mu}, q_{V}, q_{A}\right)$ arising from the chiral lagrangian $\mathcal{L}_{\text {chir }}^{(2)}$. The use of the spurions $q_{V}, q_{A}$ as sources gives rise to a simple diagrammatic method which we will in this section and the following ones. As there were some errors reported in related calculations in the past (see e.g. [36]) and we will, in fact, find some discrepancies with BU, we will present our calculations in some detail. Here, we will compute the functions $\chi_{i}\left(M_{\gamma}, \mu\right)$ which appear in eqs. (2.16). They arise from sets of one-loop diagrams containing one photon line and one pion line, the vertices being obtained from the leading order lagrangian (2.10). The corresponding standard $\xi$-gauge massive photon propagator reads

$$
D_{F}^{\mu \nu}(p)=\frac{-i}{\left(p^{2}-M_{\gamma}^{2}\right)}\left\{g^{\mu \nu}+(\xi-1) \frac{p^{\mu} p^{\nu}}{\left(p^{2}-\xi M_{\gamma}^{2}\right)}\right\} .
$$

The list of the vertices which are effectively needed is displayed in the appendix. We remark here that these contributions are to be included both in the chiral expansion of the correlator as well as in the expression from the resonance lagrangian since the latter includes the piece from eq. (2.10).

Let us now compute the contribution to $\left\langle A A Q_{A} Q_{A}\right\rangle_{i}$. A single diagram contributes, shown below

$$
+
$$


The following conventions are used to represent the sources

$$
\begin{array}{ll}
\bullet=q_{A} \text { source } & +=a_{\mu} \text { source } \\
\circ=q_{V} \text { source } & \mathrm{X}=v_{\mu} \text { source }
\end{array}
$$

The relevant vertices are listed in the appendix. One must keep in mind, for instance, that the coupling of one axial source to the pion does not contribute to our correlators. The photon line which joins the two $q$-sources is not explicitly drawn. Each diagram is easy to interpret in terms of the integral representation eq. (2.5). We will first give the results in integral form, in order to display the integrands, in which we perform the replacement of $p_{\alpha} p_{\beta}$ by $p^{2} g_{\alpha \beta} / d$. In integral form, this diagrams gives

$$
\left\langle A A Q_{A} Q_{A}\right\rangle_{1}=-F_{0}^{2} \int \frac{-i d^{d} p}{(2 \pi)^{d}} \frac{\xi}{p^{2}\left(p^{2}-\xi M_{\gamma}^{2}\right)}
$$

and the other components $\left\langle A A Q_{A} Q_{A}\right\rangle_{i}$ get no contributions. Next, we consider $\left\langle V V Q_{V} Q_{V}\right\rangle_{1}$ and, again, there is a single diagram to compute shown below

$$
\prod_{+b}^{+0}
$$

which gives

$$
\left\langle A A Q_{V} Q_{V}\right\rangle_{1}=-F_{0}^{2} \int \frac{-i d^{d} p}{(2 \pi)^{d}}\left\{\frac{1}{p^{2}\left(p^{2}-M_{\gamma}^{2}\right)}+\frac{1}{d} \frac{\xi-1}{\left(p^{2}-M_{\gamma}^{2}\right)\left(p^{2}-\xi M_{\gamma}^{2}\right)}\right\}
$$

and the other components vanish. One also finds that $\left\langle V V Q_{V} Q_{V}\right\rangle_{i}$ receive no contribution from pions. Next we consider $\left\langle V V Q_{A} Q_{A}\right\rangle_{i}$, in this case there are four diagrams
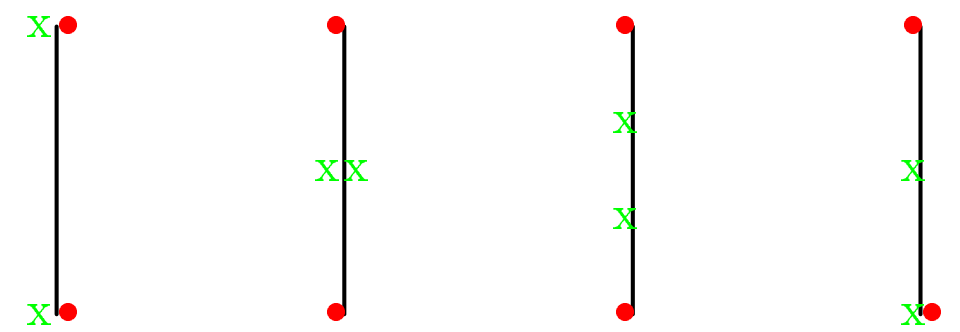

Calculating these diagrams, one finds that the last two cancel each other and the first two give the following integral

$$
\left\langle V V Q_{A} Q_{A}\right\rangle_{1}=F_{0}^{2}\left(1-\frac{1}{d}\right) \int \frac{-i d^{d} p}{(2 \pi)^{d}} \frac{\xi-1}{\left(p^{2}-M_{\gamma}^{2}\right)\left(p^{2}-\xi M_{\gamma}^{2}\right)} .
$$

Finally, we have to compute $\left\langle A V Q_{A} Q_{V}\right\rangle_{i}$. There are four diagrams in this case 

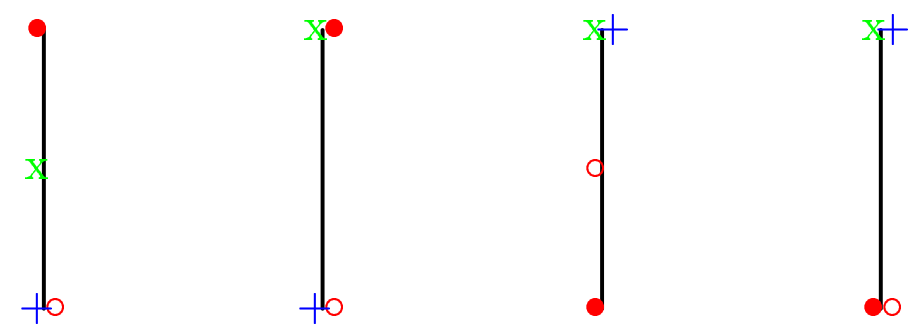

Computing these diagrams gives

$$
\begin{gathered}
\left\langle A V Q_{A} Q_{V}\right\rangle_{1}=-F_{0}^{2} \int \frac{-i d^{d} p}{(2 \pi)^{d}}\left\{\frac{1}{d} \frac{\xi}{p^{2}\left(p^{2}-\xi M_{\gamma}^{2}\right)}-\frac{1}{2} \frac{1}{p^{2}\left(p^{2}-M_{\gamma}^{2}\right)}-\right. \\
\left.-\frac{1}{2 d} \frac{\xi-1}{\left(p^{2}-M_{\gamma}^{2}\right)\left(p^{2}-\xi M_{\gamma}^{2}\right)}\right\} \\
\left\langle A V Q_{A} Q_{V}\right\rangle_{4}=\frac{F_{0}^{2}(1-d)}{k^{2}} \int \frac{-i d^{d} p}{(2 \pi)^{d}} \frac{1}{p^{2}}+\left\langle A V Q_{A} Q_{V}\right\rangle_{1} .
\end{gathered}
$$

The $1 / k^{2}$ term is generated by the third and fourth diagrams and the integral vanishes in dimensional regularization. We can now compute the d-dimensional integrals and the divergences are removed using the chiral $\overline{M S}$ prescription [3]. The results for the loop functions $\chi_{i}\left(M_{\gamma}, \mu\right)$ are collected below

$$
\begin{aligned}
& \chi_{1}\left(M_{\gamma}, \mu\right)=\frac{1}{16 \pi^{2}}\left[\xi \log \frac{M_{\gamma}^{2}}{\mu^{2}}+\xi \log \xi\right] \\
& \chi_{2}\left(M_{\gamma}, \mu\right)=\frac{1}{16 \pi^{2}}\left(\frac{1}{8}\right)\left[2(\xi+3) \log \frac{M_{\gamma}^{2}}{\mu^{2}}+2 \xi \log \xi-\xi+1\right] \\
& \chi_{3}\left(M_{\gamma}, \mu\right)=\frac{1}{16 \pi^{2}}\left(-\frac{1}{8}\right)\left[6(\xi-1) \log \frac{M_{\gamma}^{2}}{\mu^{2}}+6 \xi \log \xi+\xi-1\right] \\
& \chi_{4}\left(M_{\gamma}, \mu\right)=\frac{1}{16 \pi^{2}}\left(\frac{1}{16}\right)\left[2(\xi-3) \log \frac{M_{\gamma}^{2}}{\mu^{2}}+2 \xi \log \xi-\xi-1\right] .
\end{aligned}
$$

Using these results and those from the preceding section which display the contributions involving the chiral couplings $K_{i}^{r}$ we can deduce the scale and gauge dependence of the latter. One finds that $K_{1}^{r}, \ldots, K_{6}^{r}$ are independent of the gauge parameter $\xi$ and the scale dependence is in agreement with the results of [田, 37]. The couplings $K_{12}^{r}, K_{13}^{r}, K_{14}^{r}$ do depend on $\xi$. Defining the beta functions $\Sigma_{i}$ in the conventional manner

$$
\frac{d K_{i}^{r}(\mu)}{d \mu} \equiv-\frac{1}{16 \pi^{2}} \Sigma_{i}
$$

one finds from eqs. (2.29)

$$
\Sigma_{12}=\frac{1}{4}(3-2 \xi), \quad \Sigma_{13}=\frac{3}{4}(1-\xi), \quad \Sigma_{14}=-\frac{3}{8}(1-\xi) .
$$

These results agree with those of Urech in the special case $\xi=1$, and they reproduce the results presented in ref. [31] where a different set of correlation functions were employed. 


\section{Correlators from the minimal resonance lagrangian}

\section{1 $O\left(p^{4}\right)$ resonance lagrangian}

Our starting point for determining the $K_{i}^{r}$ parameters is the integral representation eq. (2.5) in terms of 4-point correlation functions. The first approximation that we make is to consider the leading large $N_{c}$ limit. In this limit, it is an exact statement that QCD correlators can be expressed as a sum over tree graphs involving resonance propagators [33 (see e.g. [38] for a review of recent applications). The second approximation that we make is to restrict ourselves to contributions from a finite number of resonances. This is acceptable in our case because we consider combinations of correlators which fall off as powers for large momenta.

In order to guarantee the validity of chiral Ward identities one may compute the correlators starting from a chiral lagrangian with resonances. In cases where the integrals can be shown to converge, imposing the convergence property generates a set of constraints for the parameters of the resonance lagrangian which we will call consistency conditions. These conditions will be discussed in detail in the following. We begin with the resonance lagrangian which was used by Baur and Urech [29]

$$
\mathcal{L}_{\text {res }}^{(4)}=\mathcal{L}_{\text {chir }}^{p^{2}}+\mathcal{L}_{S, S_{0}}^{(4)}+\mathcal{L}_{V, A}^{(4)} .
$$

BU have used exactly the lagrangian proposed by Ecker et al. [34] (except for leaving out the $\pi^{\prime}$ resonance multiplet) which we will call the minimal resonance lagrangian. Let us recall the notation and formalism used in ref. [34]. The formalism that is used is one in which all resonances transform homogeneously under the non linear representation of the chiral group including the vector and the axial-vector resonances which are described as antisymmetric tensor fields. In this approach, all the resonance fields can be assigned a chiral order equal to two, such that the resonance lagrangian of ref. [34, contains all possible terms, for each resonance field, which are of chiral order four. The only terms of chiral order six which are present are the kinetic energy terms.

We recall here the details: in the scalar sector an octet $S$ and a singlet $S_{0}$ are considered and the lagrangian involves two coupling-constants, $c_{d}$ and $\tilde{c}_{d}$,

$$
\begin{aligned}
\mathcal{L}_{S, S_{0}}= & \frac{1}{2}\left\langle\nabla^{\lambda} S \nabla_{\lambda} S-M_{S}^{2} S^{2}\right\rangle+\frac{1}{2}\left[\partial^{\lambda} S_{0} \partial_{\lambda} S_{0}-M_{S_{0}}^{2} S_{0}^{2}\right]+ \\
& +c_{d}\left\langle S u_{\mu} u^{\mu}\right\rangle+\tilde{c}_{d} S_{0}\left\langle u_{\mu} u^{\mu}\right\rangle .
\end{aligned}
$$

Next, a nonet of vector and axial-vector resonances are considered and the minimal lagrangian involves three coupling constants $F_{A}, F_{V}$ and $G_{V}$,

$$
\begin{aligned}
\mathcal{L}_{V, A}= & -\frac{1}{2} \sum_{R=V, A}\left\langle\nabla^{\lambda} R_{\lambda \mu} \nabla_{\nu} R^{\nu \mu}-\frac{1}{2} M_{R}^{2} R_{\mu \nu} R^{\mu \nu}\right\rangle+ \\
& +\frac{F_{A}}{2 \sqrt{2}}\left\langle A_{\mu \nu} f_{-}^{\mu \nu}\right\rangle+\frac{F_{V}}{2 \sqrt{2}}\left\langle V_{\mu \nu} f_{+}^{\mu \nu}\right\rangle+\frac{i G_{V}}{2 \sqrt{2}}\left\langle V_{\mu \nu}\left[u^{\mu}, u^{\nu}\right]\right\rangle .
\end{aligned}
$$

We will compute the basic correlators introduced in section1 first from this minimal resonance lagrangian. It is convenient to keep using the $q_{V}, q_{A}$ source spurions at this level, 
introduced in association with the $v_{\mu}$ and $a_{\mu}$ sources, with the replacements $l_{\mu} \rightarrow l_{\mu}+q_{L} F_{\mu}$, $r_{\mu} \rightarrow r_{\mu}+q_{R} F_{\mu}$. The diagrammatic calculation is exactly equivalent to using the convolution representation eq. (2.5) in which the QCD correlator is computed from the resonance lagrangian. In principle, we should be able to reproduce the results of $\mathrm{BU}$ (who used a completely different approach to determine the $K_{i}^{r}$ 's). We follow their convention to regularize the UV divergences in the chiral $\overline{M S}$ prescription and we introduce a regularization scale $\Lambda$. The various vertices needed in the calculation are collected in the appendix. We will use the photon propagator in a general $\xi$ gauge as in eq. (2.24). Since the resonances are massive one can set the photon mass $M_{\gamma}=0$ in the following. Since the $O\left(p^{2}\right)$ chiral lagrangian is also part of the resonance lagrangian, the pion contributions $\chi_{i}\left(M_{\gamma}, \Lambda\right)$, $i=1,2,3,4$ which we already computed (eqs. (2.29)) must be added to the resonance contributions.

\subsection{Contributions proportional to $F_{A}^{2}$ and $F_{V}^{2}$}

We will now consider successively the contributions proportional to $F_{A}^{2}, F_{V}^{2}, G_{V} F_{V}, G_{V}^{2}$, $G_{V}^{2} F_{V}^{2}$ which are the only ones generated from the vector and axial-vector part of the resonance lagrangian eq. (3.1). One easily sees, at first, that the following correlator components $\left\langle A A Q_{A} Q_{A}\right\rangle_{i}$ and $\left\langle V V Q_{V} Q_{V}\right\rangle_{1}$ get no contributions proportional to $F_{A}^{2}$. Next, one finds that there are five diagrams which contribute to $\left\langle V V Q_{A} Q_{A}\right\rangle_{i}$, the relevant vertices can be found in appendix A. The diagram shown below is found to make a vanishing contribution

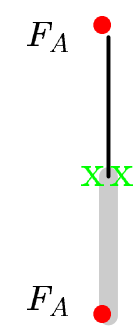

as it is antisymmetric in the $\alpha \beta$ indices. The remaining four diagrams are
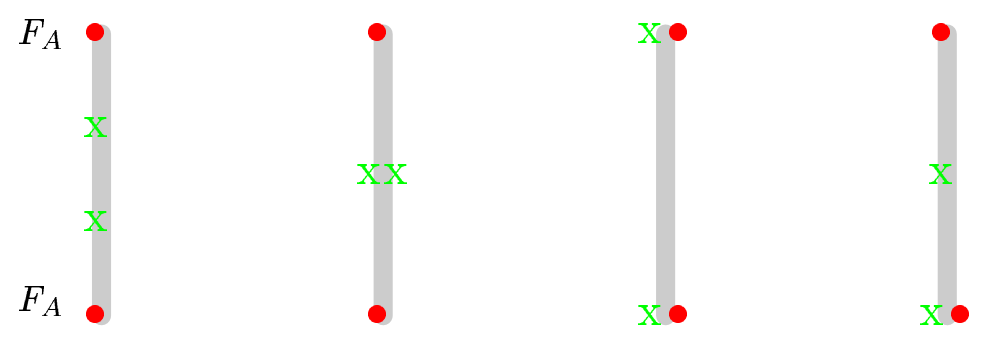

The result is of the following form

$$
\left\langle V V Q_{A} Q_{A}\right\rangle_{1}=F_{A}^{2} \int \frac{-i d^{d} p}{(2 \pi)^{d}} \sum_{1}^{4} D_{i}(p)
$$


with the individual diagrams contributions to the integrand reading

$$
\begin{aligned}
& D_{1}=\left(1-\frac{1}{d}\right)\left(\frac{2-d}{M_{A}^{2}\left(p^{2}-M_{A}^{2}\right)}+\frac{6-d}{\left(p^{2}-M_{A}^{2}\right)^{2}}+\frac{4 M_{A}^{2}}{\left(p^{2}-M_{A}^{2}\right)^{3}}\right) \\
& D_{2}=\left(1-\frac{1}{d}\right) \frac{-2}{\left(p^{2}-M_{A}^{2}\right)^{2}} \\
& D_{3}=\left(1-\frac{1}{d}\right)\left(\frac{2-d}{M_{A}^{2}\left(p^{2}-M_{A}^{2}\right)}+\frac{d-1+\xi}{p^{2}\left(p^{2}-M_{A}^{2}\right)}\right) \\
& D_{4}=\left(1-\frac{1}{d}\right)\left(\frac{2(d-2)}{M_{A}^{2}\left(p^{2}-M_{A}^{2}\right)}-\frac{4}{\left(p^{2}-M_{A}^{2}\right)^{2}}\right),
\end{aligned}
$$

respectively. Next, we consider $\left\langle A A Q_{V} Q_{V}\right\rangle$. A single diagram contributes (with a crossed one) which gives the same result as $D_{3}$ above. Finally, we turn to $\left\langle A V Q_{A} Q_{V}\right\rangle$. There are three diagrams which contribute (and no crossed diagrams in this case), which are shown below.

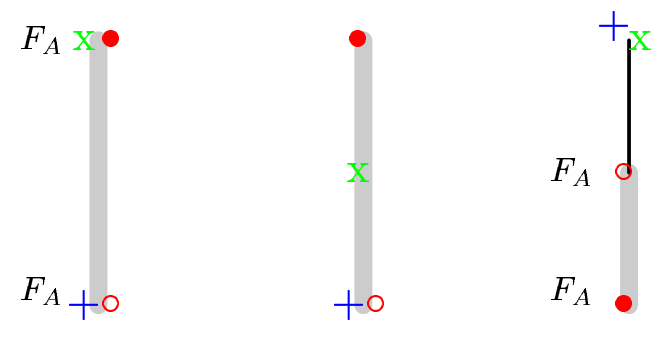

These diagrams give

$$
\begin{aligned}
\left\langle A V Q_{A} Q_{V}\right\rangle_{1} & =F_{A}^{2}\left(1-\frac{1}{d}\right) \int \frac{-i d^{d} p}{(2 \pi)^{d}}\left[\frac{d+\xi-1}{2 p^{2}\left(p^{2}-M_{A}^{2}\right)}-\frac{1}{\left(p^{2}-M_{A}^{2}\right)^{2}}\right] \\
k^{2}\left\langle A V Q_{A} Q_{V}\right\rangle_{4} & =F_{A}^{2} \int \frac{-i d^{d} p}{(2 \pi)^{d}} \frac{1-d}{p^{2}-M_{A}^{2}} .
\end{aligned}
$$

Computing all the integrals, in summary, the following results are obtained

$$
\begin{aligned}
\left\langle A A Q_{A} Q_{A}\right\rangle_{i}^{F_{A}^{2}} & =0 \\
\left\langle A A Q_{V} Q_{V}\right\rangle_{1}^{F_{A}^{2}} & =-\frac{3}{4} \frac{F_{A}^{2}}{16 \pi^{2}}(1+\xi)\left(\frac{1}{6}+\log \frac{M_{A}^{2}}{\Lambda^{2}}\right) \\
\left\langle V V Q_{A} Q_{A}\right\rangle_{1}^{F_{A}^{2}} & =\frac{3}{2} \frac{F_{A}^{2}}{16 \pi^{2}}\left\{1-\frac{1}{2}(\xi-1)\left(\frac{1}{6}+\log \frac{M_{A}^{2}}{\Lambda^{2}}\right)\right\} \\
\left\langle V V Q_{V} Q_{V}\right\rangle_{1}^{F_{A}^{2}} & =0 \\
\left\langle A V Q_{A} Q_{V}\right\rangle_{1}^{F_{A}^{2}} & =-\frac{3}{8} \frac{F_{A}^{2}}{16 \pi^{2}}(1+\xi)\left(\frac{1}{6}+\log \frac{M_{A}^{2}}{\Lambda^{2}}\right) \\
k^{2}\left\langle A V Q_{A} Q_{V}\right\rangle_{4}^{F_{A}^{2}} & =3 \frac{M_{A}^{2} F_{A}^{2}}{16 \pi^{2}}\left(\frac{2}{3}+\log \frac{M_{A}^{2}}{\Lambda^{2}}\right)+O\left(k^{2}\right) .
\end{aligned}
$$

There is a simple correspondence between the diagrams proportional to $F_{V}^{2}$ and those proportional to $F_{A}^{2}$ considered above so we give directly the results for the various components 
of the correlators

$$
\begin{aligned}
\left\langle A A Q_{A} Q_{A}\right\rangle_{1}^{F_{V}^{2}} & =-\frac{3}{4} \frac{F_{V}^{2}}{16 \pi^{2}}(1+\xi)\left(\frac{1}{6}+\log \frac{M_{V}^{2}}{\Lambda^{2}}\right) \\
\left\langle A A Q_{V} Q_{V}\right\rangle_{i}^{F_{V}^{2}} & =0 \\
\left\langle V V Q_{A} Q_{A}\right\rangle_{1}^{F_{V}^{2}} & =0 \\
\left\langle V V Q_{V} Q_{V}\right\rangle_{1}^{F_{V}^{2}} & =\frac{3}{2} \frac{F_{V}^{2}}{16 \pi^{2}}\left\{1-\frac{1}{2}(\xi-1)\left(\frac{1}{6}+\log \frac{M_{V}^{2}}{\Lambda^{2}}\right)\right\} \\
\left\langle A V Q_{A} Q_{V}\right\rangle_{1}^{F_{V}^{2}} & =-\frac{3}{8} \frac{F_{V}^{2}}{16 \pi^{2}}(1+\xi)\left(\frac{1}{6}+\log \frac{M_{V}^{2}}{\Lambda^{2}}\right) \\
k^{2}\left\langle A V Q_{A} Q_{V}\right\rangle_{4}^{F_{V}^{2}} & =-3 \frac{M_{V}^{2} F_{V}^{2}}{16 \pi^{2}}\left(\frac{2}{3}+\log \frac{M_{V}^{2}}{\Lambda^{2}}\right) .
\end{aligned}
$$

\section{$3.3 G_{V} F_{V}$ contributions}

We have two diagrams which contribute to $\left\langle A A Q_{A} Q_{A}\right\rangle_{1}$ shown below.
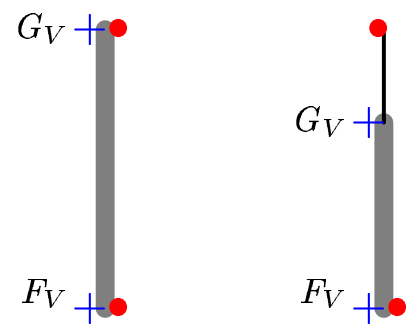

This results in the following integral for the correlator

$$
\left\langle A A Q_{A} Q_{A}\right\rangle_{1}=4 G_{V} F_{V}\left(1-\frac{1}{d}\right) \int \frac{-i d^{d} p}{(2 \pi)^{d}}\left(\frac{d-2}{M_{V}^{2}\left(p^{2}-M_{V}^{2}\right)}+\frac{1-d}{p^{2}\left(p^{2}-M_{V}^{2}\right)}\right) .
$$

Next we have one diagram contributing to $\left\langle A A Q_{V} Q_{V}\right\rangle_{1}$ which is non vanishing

$$
\begin{gathered}
\left.G_{V}+\right]^{\circ} \\
F_{V}
\end{gathered}
$$

and which gives

$$
\left\langle A A Q_{V} Q_{V}\right\rangle_{1}=4 G_{V} F_{V} \int \frac{-i d^{d} p}{(2 \pi)^{d}} \frac{1}{p^{2}\left(p^{2}-M_{V}^{2}\right)} .
$$

The two correlators $\left\langle V V Q_{A} Q_{A}\right\rangle_{1}$ and $\left\langle V V Q_{V} Q_{V}\right\rangle_{1}$ are then found to get no contributions proportional to $G_{V} F_{V}$.

Next, we consider $\left\langle A V Q_{A} Q_{V}\right\rangle_{i}$. One first finds a set of 7 diagrams which contribute both to $\left\langle A V Q_{A} Q_{V}\right\rangle_{1}$ and $\left\langle A V Q_{A} Q_{V}\right\rangle_{4}$. The three diagrams drawn below give zero contribution 


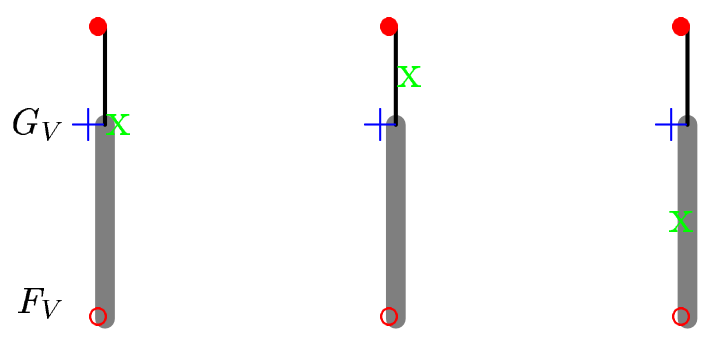

The next four diagrams are
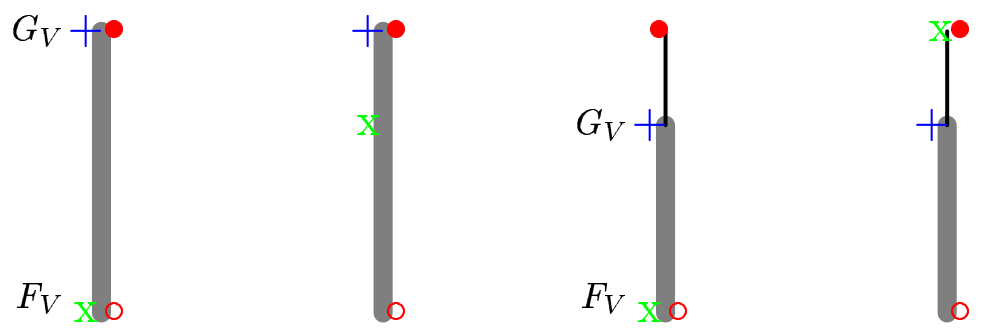

Computing these four diagrams yields the following result for $\left\langle A V Q_{A} Q_{V}\right\rangle_{1}$

$$
\left\langle A V Q_{A} Q_{V}\right\rangle_{1}=G_{V} F_{V}\left(1-\frac{1}{d}\right) \int \frac{-i d^{d} p}{(2 \pi)^{d}}\left(\frac{-d}{p^{2}\left(p^{2}-M_{V}^{2}\right)}+\frac{2}{\left(p^{2}-M_{V}^{2}\right)^{2}}\right),
$$

these diagrams make an identical contribution also to $\left\langle A V Q_{A} Q_{V}\right\rangle_{4}$. In order to get the full contribution to $\left\langle A V Q_{A} Q_{V}\right\rangle_{4}$ we must also add the following two diagrams

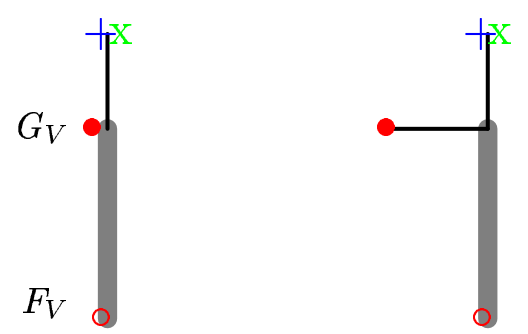

These diagrams are slightly more complicated to compute because one must allow for a small momentum to flow through the $v_{\beta}$ source and expand the result in powers of $k^{2}$. One finds that the $k^{2}$ pole vanishes and then adding the constant contribution to that arising from the preceding four diagrams one finds that they cancel. In summary, the contributions proportional to $G_{V} F_{V}$ which are non vanishing read

$$
\begin{aligned}
\left\langle A A Q_{A} Q_{A}\right\rangle_{1}^{G_{V} F_{V}} & =\frac{3 G_{V} F_{V}}{16 \pi^{2}}\left(\frac{1}{6}+\log \frac{M_{V}^{2}}{\Lambda^{2}}\right) \\
\left\langle A A Q_{V} Q_{V}\right\rangle_{1}^{G_{V} F_{V}} & =-\frac{3 G_{V} F_{V}}{16 \pi^{2}}\left(\frac{1}{6}+\log \frac{M_{V}^{2}}{\Lambda^{2}}\right) \\
\left\langle A V Q_{A} Q_{V}\right\rangle_{1}^{G_{V} F_{V}} & =\frac{3}{2} \frac{G_{V} F_{V}}{16 \pi^{2}}\left(\frac{1}{6}+\log \frac{M_{V}^{2}}{\Lambda^{2}}\right) .
\end{aligned}
$$




\section{4 $G_{V}^{2}$ and $G_{V}^{2} F_{V}^{2}$ contributions}

These contributions are simple because a single correlator component is found to be non vanishing: $\left\langle A A Q_{A} Q_{A}\right\rangle_{1}$ in the case of $G_{V}^{2}$ and $\left\langle A A Q_{V} Q_{V}\right\rangle_{1}$ in the case of $G_{V}^{2} F_{V}^{2}$. There are three diagrams proportional to $G_{V}^{2}$
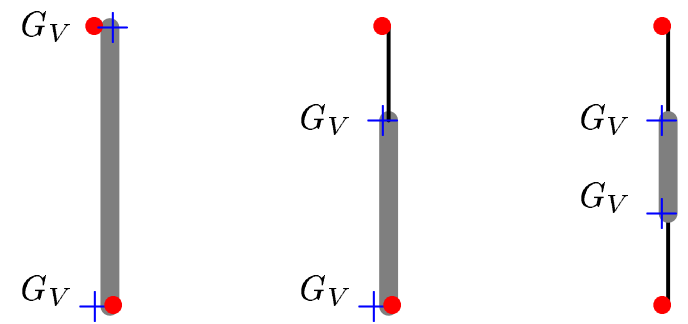

and a single one proportional to $G_{V}^{2} F_{V}^{2}$

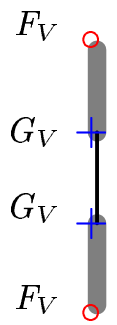

After a small computation the following results are obtained,

$$
\begin{aligned}
& \left\langle A A Q_{A} Q_{A}\right\rangle_{1}=4 G_{V}^{2}\left(1-\frac{1}{d}\right) \int \frac{-i d^{d} p}{(2 \pi)^{d}}\left(\frac{2-d}{M_{V}^{2}\left(p^{2}-M_{V}^{2}\right)}+\frac{d-1}{p^{2}\left(p^{2}-M_{V}^{2}\right)}\right) \\
& \left\langle A A Q_{V} Q_{V}\right\rangle_{1}=-4 \frac{G_{V}^{2} F_{V}^{2}}{F_{0}^{2}}\left(1-\frac{1}{d}\right) \int \frac{-i d^{d} p}{(2 \pi)^{d}} \frac{1}{\left(p^{2}-M_{V}^{2}\right)^{2}} .
\end{aligned}
$$

In summary, the non vanishing contributions proportional to $G_{V}^{2}$ read

$$
\begin{aligned}
\left\langle A A Q_{A} Q_{A}\right\rangle_{1}^{G_{V}^{2}} & =-\frac{3 G_{V}^{2}}{16 \pi^{2}}\left(\frac{1}{6}+\log \frac{M_{V}^{2}}{\Lambda^{2}}\right) \\
\left\langle A A Q_{V} Q_{V}\right\rangle_{1}^{G_{V}^{2} F_{V}^{2}} & =\frac{3 G_{V}^{2} F_{V}^{2}}{16 \pi^{2} F_{0}^{2}}\left(\frac{7}{6}+\log \frac{M_{V}^{2}}{\Lambda^{2}}\right) .
\end{aligned}
$$

\subsection{Contributions proportional to $c_{d}, \tilde{c}_{d}$}

The scalar singlet turns out to contribute only to $\left\langle A A Q_{A} Q_{A}\right\rangle_{2}$ and the scalar octet to $\left\langle A A Q_{A} Q_{A}\right\rangle_{3}$. The three Feynman diagrams to be computed are depicted below.
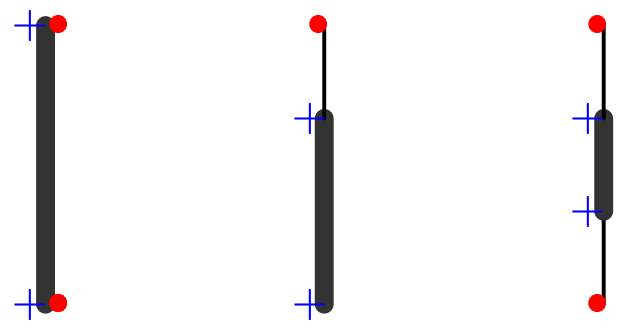
The dependence on the photon gauge parameter $\xi$ is found to cancel out, and the following results are obtained for the singlet and octet scalar contributions

$$
\begin{aligned}
& \left\langle A A Q_{A} Q_{A}\right\rangle_{2}=16 \tilde{c}_{d}^{2}\left(1-\frac{1}{d}\right) \int \frac{-i d^{d} p}{(2 \pi)^{d}} \frac{1}{p^{2}\left(M_{S_{0}}^{2}-p^{2}\right)} \\
& \left\langle A A Q_{A} Q_{A}\right\rangle_{3}=8 c_{d}^{2}\left(1-\frac{1}{d}\right) \int \frac{-i d^{d} p}{(2 \pi)^{d}} \frac{1}{p^{2}\left(M_{S}^{2}-p^{2}\right)} .
\end{aligned}
$$

As before, we have replaced the term $p_{\alpha} p_{\beta}$ in the integrands by $p^{2} g_{\alpha \beta} / d$. There are three additional tadpole diagrams. Let us quote the result for completeness

$$
\left\langle A_{\alpha}^{a} A_{\beta}^{b} Q_{A}^{c} Q_{A}^{d}\right\rangle^{t a d}=i g_{\alpha \beta}\left(\frac{8 c_{d}^{2}}{M_{S}^{2}} d^{s a b} d^{s c d}+\frac{16 \tilde{c}_{d}^{2}}{M_{S_{0}}^{2}} \delta^{a b} \delta^{c d}\right) \int \frac{-i d^{d} p}{(2 \pi)^{d}} \frac{3}{p^{2}} .
$$

Computing the integrals we obtain the following results for the scalar resonance contributions which are non vanishing

$$
\begin{aligned}
\left\langle A A Q_{A} Q_{A}\right\rangle_{2}^{\text {scal }} & =12 \frac{\tilde{c}_{d}^{2}}{16 \pi^{2}}\left(\log \frac{M_{S_{0}}^{2}}{\Lambda^{2}}+\frac{1}{6}\right) \\
\left\langle A A Q_{A} Q_{A}\right\rangle_{3}^{\text {scal }} & =6 \frac{c_{d}^{2}}{16 \pi^{2}}\left(\log \frac{M_{S}^{2}}{\Lambda^{2}}+\frac{1}{6}\right) .
\end{aligned}
$$

One notices that no dependence upon the gauge parameter $\xi$ appears except in some of the terms proportional to $F_{A}^{2}$ and $F_{V}^{2}$. This is to be expected because, quite generally, the part proportional to $\xi$ in the integral representation can be simplified by making use of chiral Ward identities and expressed in terms of 2-point Green's functions [31].

\subsection{Comparison with the results of Baur and Urech}

Up to this point we have used exactly the same resonance lagrangian terms as Baur and Urech [29] and we are therefore in a position to check their results for the chiral parameters $K_{i}^{r}$. Making use of the relations (2.14) 2.18), for each resonance term contribution to the correlators (2.3) (2.4) we can solve for the $K_{i}^{r}$ 's which will then depend on the two scales $\mu$ and $\Lambda$. In ref. 29] the values of the two scales have been taken to be equal. Following this convention for the sake of comparison, and using the results derived above, we reproduce the results of ref. 29] for the parameters $K_{1}^{r}, \ldots, K_{6}^{r}$.

We disagree, however, on a number of contributions which concern $K_{12}^{r}, K_{13}^{r}, K_{14}^{r}$. Contrary to BU, we find that the following quantities are vanishing.

$$
K_{13}^{G_{V} F_{V}}=K_{14}^{G_{V} F_{V}}=K_{12}^{G_{V}^{2}}=K_{13}^{G_{V}^{2}}=K_{14}^{G_{V}^{2}}=K_{12}^{G_{V}^{2} F_{V}^{2}}=K_{13}^{G_{V}^{2} F_{V}^{2}}=K_{14}^{G_{V}^{2} F_{V}^{2}}=0 .
$$

In the case of $K_{13}^{r}, K_{14}^{r}$ this follows from [31] where it is shown that they can be obtained from 2-point correlators $\left\langle Q_{V} Q_{V}\right\rangle$ and $\left\langle Q_{A} Q_{A}\right\rangle$. Obviously, these can only pick up contributions proportional to $F_{A}^{2}$ and $F_{V}^{2}$, which is indeed what we recover here. Similarly, one can also obtain $K_{12}^{r}$ from a correlator with three currents $\left\langle P Q_{A} Q_{V}\right\rangle$ which picks up no contribution proportional to $G_{V}^{2}$. 

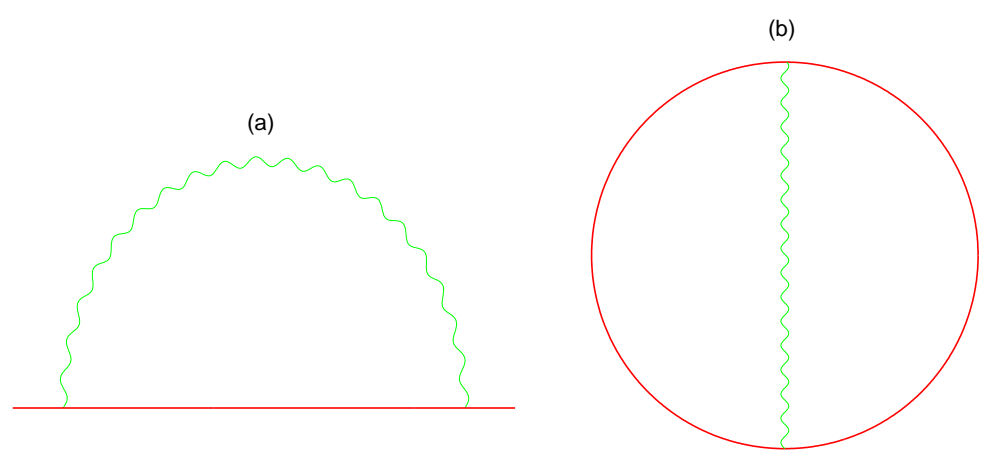

Figure 1: Typical divergent diagrams quadratic in the spurion sources $(a)$ with an open quark line (b) with a closed quark line.

\section{Short distance constraints}

We now need to determine the divergence structure in the integral representation of eq. (2.5) according to QCD. One way to proceed is to determine the necessary counterterms which are generated in QCD with a source term as in eq. (2.1), where $F_{\mu}$ is a dynamical (massive) photon. This QED-like sector differs from ordinary QED by the fact that we have replaced the charge matrix $Q$ by a vector $\left(q_{V}\right)$ as well as an axial-vector $\left(q_{A}\right)$ spurion source. We must consider this theory as a kind of effective theory and we assume that the divergences can be absorbed into a set of local lagrangian terms. We will restrict ourselves to an expansion quadratic in the spurion fields. This represents an important simplification since, for instance, we will only need the free photon propagator and thereby avoid the problems of the photon propagator at higher order in the presence of axial couplings [39]. Another restriction is that we will only consider contributions which are of leading order in $N_{c}$. Dimensional regularization together with the naive $\gamma_{5}$ prescription will be used.

\subsection{QCD+QED counterterms}

Let us consider QCD in the presence of dynamical photons and a source term of the form of eq. (2.1). The effective action is expanded to quadratic order in the spurions $q_{V}$ and $q_{A}$ and we drop the contributions which are subleading in $N_{c}$. We can then split the divergent terms in the effective action into two categories, $(a)$ one which collects the graphs with an open quark line and, $(b)$ one which collects the graphs with a closed quark loop (see figure (1). The former ones are those which will prove to be of relevance to us.

Let us first consider the contributions in category $(b)$. The graphs in this category, at any order in $\alpha_{s}$ (in QCD the three gluon vertex has dimension $p$, the four gluon vertex has dimension zero), have dimensionality $p^{4}$. The corresponding local counterterms therefore must involve four derivatives. Examples of such terms are,

$$
\left\langle\nabla^{2} q_{L} \nabla^{2} q_{L}+\nabla^{2} q_{R} \nabla^{2} q_{R}\right\rangle, \quad G_{\mu \nu} G^{\mu \nu}\left\langle\nabla_{\rho} q_{L} \nabla^{\rho} q_{L}+\nabla_{\rho} q_{R} \nabla^{\rho} q_{R}\right\rangle, \quad \text { etc... }
$$

If we restrict ourselves to the correlator combinations $\Pi_{i}$, eq. (2.19) (from which the contribution proportional to $K_{14}$ drops out) then all the contributions from such counterterms also drop out or are suppressed in the large $N_{c}$ counting. 
We are finally left with the contributions from the first category. Taking into account the structure of QCD, all graphs in this category have dimension $p$, implying that the counterterms must involve one derivative. Furthermore, they must involve two spurions of the same kind (either $q_{L}$ or $q_{R}$ ). One can form only two such independent terms

$$
\begin{aligned}
\mathcal{L}= & \frac{i}{2} Z_{2}\left\{\bar{\psi}_{L}\left[q_{L}, \nabla^{\mu} q_{L}\right] \gamma_{\mu} \psi_{L}+\bar{\psi}_{R}\left[q_{R}, \nabla^{\mu} q_{R}\right] \gamma_{\mu} \psi_{R}\right\}+ \\
& +\frac{i}{2} Z_{2}^{\prime}\left\{\bar{\psi}_{L} q_{L}^{2} \gamma_{\mu} D^{\mu} \psi_{L}+\bar{\psi}_{R} q_{R}^{2} \gamma_{\mu} D^{\mu} \psi_{R}+\text { h.c. }\right\}
\end{aligned}
$$

where

$$
i D^{\mu} \psi_{L}=\left(i \partial^{\mu}+l_{\mu}+G_{\mu}\right) \psi_{L}, \quad i D^{\mu} \psi_{R}=\left(i \partial^{\mu}+r_{\mu}+G_{\mu}\right) \psi_{R} .
$$

At order zero in the strong coupling constant one has the well known QED result

$$
Z_{2}=Z_{2}^{\prime}=\frac{\mu_{0}^{-2 \epsilon}}{16 \pi^{2}}(-\xi)[\Gamma(\epsilon)+\log (4 \pi)]+O\left(\alpha_{s}\right) .
$$

Equality between $Z_{2}$ and $Z_{2}^{\prime}$ need not hold beyond the lowest order.

Let us now discuss the contributions proportional to $Z_{2}$ and $Z_{2}^{\prime}$ to our basic correlators (2.3) (2.4). The first result is that the contribution proportional to $Z_{2}^{\prime}$ vanishes at leading order in $N_{c}$. This can be shown in a pedestrian way. Because of the conservation of chirality in one fermion loop graphs, the contributions concern only the correlators $\left\langle L L Q_{L} Q_{L}\right\rangle$ and $\left\langle R R Q_{R} Q_{R}\right\rangle$, it suffices to consider the former. For a given gluon field configuration $G_{\mu}(x)$ we can express the functional derivatives with respect to the sources in terms of exact fermion propagators $S_{G}(x, y)$ and we obtain the following expression

$$
\begin{aligned}
&\left\langle L_{\alpha}^{a} L_{\beta}^{b} Q_{L}^{c} Q_{L}^{d}\right\rangle_{G}=\frac{Z_{2}^{\prime}}{32} \int d^{4} x d^{4} y[-\delta(y)\left\langle\lambda^{b} \lambda^{a}\left\{\lambda^{c}, \lambda^{d}\right\}\right\rangle\left\langle\gamma^{\alpha} P_{L} S_{G}(x, 0) \gamma^{\beta} P_{L} S_{G}(0, x)\right\rangle- \\
&-\delta(x)\left\langle\lambda^{a} \lambda^{b}\left\{\lambda^{c}, \lambda^{d}\right\}\right\rangle\left\langle\gamma^{\beta} P_{L} S_{G}(y, 0) \gamma^{\alpha} P_{L} S_{G}(0, y)\right\rangle \\
&+\left\langle\lambda^{a} \lambda^{b}\left\{\lambda^{c}, \lambda^{d}\right\}\right\rangle\left\langle\gamma^{\alpha} P_{L} S_{G}(x, y) \gamma^{\beta} P_{L} S_{G}(y, 0) \times\right. \\
& \times {\left.\left[i \vec{\phi}+G^{\prime}\right] P_{L} S_{G}(0, x)\right\rangle+ } \\
&+\left\langle\lambda^{b} \lambda^{a}\left\{\lambda^{c}, \lambda^{d}\right\}\right\rangle\left\langle\gamma^{\beta} P_{L} S_{G}(y, x) \gamma^{\alpha} P_{L} S_{G}(x, 0) \times\right. \\
&\left.\left.\times\left[i \vec{\phi}+G_{G}\right] P_{L} S_{G}(0, y)\right\rangle\right]
\end{aligned}
$$

where $P_{L}$ is the projector $P_{L}=1 / 2\left(1-\gamma_{5}\right)$ and the notation $\vec{\not}$ means that the derivatives are acting on the right. There is another similar contribution coming from the hermitian conjugate part of the lagrangian. Using the equation obeyed by the Dirac propagator

$$
\left[i \vec{\not}+\phi^{x}\right] P_{L} S_{G}(x, y)=P_{R} \delta^{4}(x-y),
$$

we indeed find that the various terms in eq. (4.5) cancel each other. We have thus shown that the only relevant contributions from the counterterms are those proportional to $Z_{2}$. The calculation of these is performed by computing the functional derivatives according to the definition of eq. (2.3) and using the following result for the two-point correlators that appear

$$
\int d^{4} x \mathrm{e}^{i k x}\left\langle A_{\alpha}^{s}(x) A_{\beta}^{t}(0)\right\rangle=i F_{0}^{2} \delta^{s t}\left(\frac{k_{\alpha} k_{\beta}}{k^{2}}-g_{\alpha \beta}\right), \quad \int d^{4} x\left\langle V_{\alpha}^{s}(x) V_{\beta}^{t}(0)\right\rangle=0 .
$$


We find that the counterterms contribute to the following components of our basic correlators

$$
\begin{aligned}
\left\langle A A Q_{A} Q_{A}\right\rangle_{1}^{c t} & =-Z_{2} F_{0}^{2} \\
\left\langle A A Q_{V} Q_{V}\right\rangle_{1}^{c t} & =-Z_{2} F_{0}^{2} \\
\left\langle A V Q_{A} Q_{V}\right\rangle_{1}^{c t} & =-\frac{1}{2} Z_{2} F_{0}^{2} \\
\left\langle A V Q_{A} Q_{V}\right\rangle_{4}^{c t} & =-\frac{1}{2} Z_{2} F_{0}^{2} .
\end{aligned}
$$

There are no contributions to the other components.

\subsection{Summary of the $\log \Lambda^{2}$ dependence}

Let us consider the combinations of correlators $\Pi_{i}$ introduced in eqs. (2.19) (2.23). In all these combinations the contribution from the coupling $K_{14}^{r}$ drops out. In the combinations $\Pi_{1}, \ldots, \Pi_{6}$ as well as $\Pi_{7}, \Pi_{9}$, the contributions from the renormalization parameter $Z_{2}$ also drops out, as is seen from using eq. (4.8). As a consequence, these quantities must be finite. More precisely, finiteness holds in the sense of dimensional regularization which was used to establish the form of the counterterms in the preceding section: one must compute the photon loop integral in $d$ dimensions and then take the limit $d \rightarrow 4$ which must be finite. From these eight combinations one can then determine the chiral couplings $K_{1}^{r}, \ldots, K_{6}^{r}$ as well as $K_{13}^{r}$ and $C$. The situation is different for $\Pi_{8}$ since, in that case, a contribution proportional to $Z_{2}$ is present. The integral is not finite in general. It is finite to zeroth order in the strong coupling $\alpha_{s}$ when the gauge parameter $\xi=0$. This limitation affects the determination of the coupling $K_{12}^{r}$.

Let us examine how these finiteness conditions are satisfied when the relevant correlators are computed from the minimal resonance lagrangian of eqs. (3.2), (3.3). Collecting the results presented in detail in section 3, we obtain the following dependence upon the regularization scale $\Lambda$

$$
\begin{aligned}
& \Pi_{1} \sim-\frac{3}{2} \frac{\log \Lambda^{2}}{16 \pi^{2}}\left[F_{0}^{2}+F_{A}^{2}-2 G_{V}^{2}\right] \\
& \Pi_{2} \sim-\frac{3}{2} \frac{\log \Lambda^{2}}{16 \pi^{2}}\left[F_{0}^{2}+F_{V}^{2}-4 G_{V} F_{V}+2 G_{V}^{2} \frac{F_{V}^{2}}{F_{0}^{2}}\right] \\
& \Pi_{3} \sim-6 \frac{\log \Lambda^{2}}{16 \pi^{2}}\left[c_{d}^{2}\right] \\
& \Pi_{4}=0 \\
& \Pi_{5} \sim-4 \frac{\log \Lambda^{2}}{16 \pi^{2}}\left[3 \tilde{c}_{d}^{2}-c_{d}^{2}\right] \\
& \Pi_{6}=0 \\
& \Pi_{7} \sim \frac{3}{4} \frac{\log \Lambda^{2}}{16 \pi^{2}}\left[F_{V}^{2}-F_{0}^{2}-F_{A}^{2}\right] \\
& \Pi_{8} \sim-\frac{3}{8} \frac{\log \Lambda^{2}}{16 \pi^{2}}\left[F_{V}^{2}-F_{0}^{2}-F_{A}^{2}+4\left(G_{V} F_{V}-F_{V}^{2}\right)\right] \\
& \Pi_{9} \sim 3 \frac{\log \Lambda^{2}}{16 \pi^{2}}\left[M_{V}^{2} F_{V}^{2}-M_{A}^{2} F_{A}^{2}\right] .
\end{aligned}
$$


At this level, there is at first one consistency condition which is satisfied, but trivially so, since $\Pi_{4}$ is identically vanishing. In the sequel (section 5.6) we will consider physically relevant interactions and attempt to satisfy the condition in a less trivial way. The consistency conditions for $\Pi_{7}$ and $\Pi_{9}$ can be satisfied and they generate the two Weinberg sum rule relations

$$
F_{V}^{2}=F_{0}^{2}+F_{A}^{2}, \quad M_{V}^{2} F_{V}^{2}=M_{A}^{2} F_{A}^{2} .
$$

These relations will be assumed to hold from now on. Furthermore, we will assume the following relation to hold,

$$
G_{V} F_{V}=F_{0}^{2}
$$

which follows 35 from imposing that the asymptotic behaviour of the vector form-factor of the pion

$$
\lim _{t \rightarrow \infty} t F_{\pi}^{V}(t)=0
$$

is satisfied. Using this, we observe that there are only two consistency conditions which are fulfilled, which are those associated with $\Pi_{7}$ and $\Pi_{9}$. The corresponding evaluation of $\Pi_{7}$ and $\Pi_{9}$ gives

$$
\begin{aligned}
& \Pi_{7}=\frac{3}{4} \frac{F_{0}^{2}}{16 \pi^{2}}\left[\log \frac{M_{V}^{2}}{M_{\gamma}^{2}}+2-\frac{\log z}{z-1}\right] \\
& \Pi_{9}=3 M_{V}^{2} \frac{F_{0}^{2}}{16 \pi^{2}} \frac{\log z}{z-1},
\end{aligned}
$$

where $z$ denotes the mass ratio

$$
z=\frac{M_{A}^{2}}{M_{V}^{2}}
$$

which one expects to be approximately equal to 2 . We expect $\Pi_{5}$ and $\Pi_{6}$ to vanish as a result of the leading large $N_{c}$ approximation. This condition is trivially satisfied for $\Pi_{6}$ and it can be imposed for $\Pi_{5}$ by setting $3 \tilde{c}_{d}^{2}=c_{d}^{2}$.

\section{Beyond the $O\left(p^{4}\right)$ resonance lagrangian}

\subsection{Survey of $O\left(p^{6}\right)$ terms}

In order to comply with the other consistency conditions, it is necessary to enlarge the resonance lagrangian of eq. (3.1). Since this lagrangian (for a given resonance content) contains all terms of order $p^{4}$, it is natural to investigate terms of order six. Generically, let us designate a resonance building block by $R$ (which counts as $p^{2}$ ), a chiral building block by $\pi$ (which counts as $p$ ) and a source term by $J$ (which counts as $p^{2}$ ). In this schematic notation, there are four classes of terms of order $p^{4}$ which involve at least one resonance

$$
R R, R J, R \pi, R \pi \pi \text {. }
$$

At order $p^{6}$ one can have the same terms but involving two more derivatives and, in addition, one can have terms formed from three, four as well as five building blocks

$$
R R R, R R \pi, R R J, R J J, R J \pi, R R \pi \pi, R J \pi \pi, R \pi \pi \pi, R \pi \pi \pi \pi \text {. }
$$


We will not attempt to classify and discuss all possible remaining terms in this work. We will simply consider (except in section 5.6) sets of terms of the form $R R \pi$. The first set will be labeled as $a_{1} \rho \pi$ and has the following form

$$
\begin{aligned}
\mathcal{L}_{a_{1} \rho \pi}= & i\left(G_{1} g^{\mu_{0} \mu_{1}} g^{\mu_{2} \mu 3}+G_{2} g^{\mu_{0} \mu_{2}} g^{\mu_{3} \mu_{1}}+G_{3} g^{\mu_{0} \mu_{3}} g^{\mu_{1} \mu_{2}}\right)\left\langle\left[\nabla_{\mu_{0}} V_{\mu_{1} \lambda}, A_{\mu_{2}}{ }^{\lambda}\right] u_{\mu_{3}}\right\rangle+ \\
& +i\left(H_{1} g^{\mu_{0} \mu_{1}} g^{\mu_{2} \mu_{3}}+H_{2} g^{\mu_{0} \mu_{2}} g^{\mu_{3} \mu_{1}}+H_{3} g^{\mu_{0} \mu_{3}} g^{\mu_{1} \mu_{2}}\right)\left\langle\left[V_{\mu_{1} \lambda}, A_{\mu_{2}}{ }^{\lambda}\right] \nabla_{\mu_{0}} u_{\mu_{3}}\right\rangle .
\end{aligned}
$$

We will first show that by adding these terms to the minimal resonance lagrangian we can reproduce the $\langle V A P\rangle$ 3-point function constructed in ref. [31]. ${ }^{1}$ In addition, we will then show that it is possible to satisfy the consistency conditions associated with $\Pi_{1}$ and $\Pi_{2}$, and these provides a number of Weinberg-type sum rule relations. In an analogous way, we introduce an $a_{0} f_{1} \pi$ coupling (we assume nonet symmetry which reduces the number of couplings),

$$
\mathcal{L}_{a_{0} f_{1} \pi}=c_{A}\left\langle S\left\{\nabla^{\mu} A_{\mu \nu}, u^{\nu}\right\}\right\rangle
$$

which will allow us to satisfy the consistency condition on $\Pi_{3}$.

Finally, we will consider a set of terms of the type $\omega \rho \pi$ which contribute to $\Pi_{4}$. A calculation which is equivalent to an evaluation of this contribution was performed in ref. [7] using the vector, instead of the anti-symmetric tensor, formulation for the vector fields. A classification of the terms in this latter formulation was recently presented by Ruiz-Femenia et al. 41], we list below those of relevance to us,

$$
\begin{aligned}
\mathcal{L}_{\omega \rho \pi}=\epsilon_{\mu \nu \rho \sigma}\{ & d_{3}\left\langle\left\{\nabla_{\lambda} V^{\mu \nu}, V^{\rho \lambda}\right\} u^{\sigma}\right\rangle+d_{4}\left\langle\left\{\nabla^{\sigma} V^{\mu \nu}, V^{\rho \lambda}\right\} u_{\lambda}\right\rangle+ \\
& +\frac{c_{5}}{M_{V}}\left\langle\left\{\nabla_{\lambda} V^{\mu \nu}, f_{+}^{\rho \lambda}\right\} u^{\sigma}\right\rangle+\frac{c_{6}}{M_{V}}\left\langle\left\{\nabla_{\lambda} V^{\mu \lambda}, f_{+}^{\rho \sigma}\right\} u^{\nu}\right\rangle+ \\
& \left.+\frac{c_{7}}{M_{V}}\left\langle\left\{\nabla^{\sigma} V^{\mu \nu}, f_{+}^{\rho \lambda}\right\} u_{\lambda}\right\rangle\right\} .
\end{aligned}
$$

It enabled them to reproduce the 3-point function $\langle V V P\rangle$ as constructed in ref. [42]. The consistency condition on $\Pi_{4}$ is somewhat more complicated to satisfy in the antisymmetric tensor than in the vector formalism. This will be explained in more detail below.

\section{$5.2 \mathcal{L}_{a_{1} \rho \pi}$ constraints from $\langle V A P\rangle$}

Let us examine the constraints on the six coupling constants of the $a_{1} \rho \pi$ lagrangian (5.3) which arise from the 3-point correlator $\langle V A P\rangle$. This correlator is defined as follows

$$
W_{\alpha \beta}(p, q)=\int d^{4} x d^{4} y \mathrm{e}^{i p x+i q y}\left\langle 0\left|T\left(V_{\alpha}^{1}(x) A_{\beta}^{2}(y) P^{3}(0)\right)\right| 0\right\rangle .
$$

Assuming an ansatz in terms of rational functions with a minimal number of resonance poles (i.e. one pion, one vector and one axial-vector resonance poles) it can be shown [31]

\footnotetext{
${ }^{1}$ An extension was recently considered [40] which includes a $\pi^{\prime}$ resonance multiplet and a corresponding resonance chiral lagrangian was constructed. The result for the coupling constant combination $G_{1}+G_{2}+2 G_{3}$ which is needed in the following turns out to be unaffected by this extension.
} 
that this correlator is uniquely determined from $(a)$ the chiral Ward identities, $(b)$ the constraint to match the leading asymptotic scaling behaviour,

$$
\lim _{\lambda \rightarrow \infty} W_{\alpha \beta}(\lambda p, \lambda q)=\frac{\langle\bar{u} u\rangle}{\lambda^{2}}\left\{\frac{\left(p_{\alpha}+2 q_{\alpha}\right) q_{\beta}-q^{2} g_{\alpha \beta}}{q^{2} l^{2}}+\frac{\left(p^{2}-q^{2}-l^{2}\right) P_{\alpha \beta}}{2 p^{2} q^{2} l^{2}}-\frac{Q_{\alpha \beta}}{p^{2} q^{2} l^{2}}\right\}
$$

with $l^{2}=(p+q)^{2}$,

$$
\begin{aligned}
P_{\alpha \beta} & =p_{\beta} q_{\alpha}-p \cdot q g_{\alpha \beta} \\
Q_{\alpha \beta} & =p^{2} q_{\alpha} q_{\beta}+q^{2} p_{\alpha} p_{\beta}-p . q p_{\alpha} q_{\beta}-p^{2} q^{2} g_{\alpha \beta},
\end{aligned}
$$

and, finally, $(c)$ the asymptotic constraint on the vector form factor of the pion eq. (4.12). More detailed analysis of asymptotic constraints related to this 3-point correlator were performed in ref. [43]. The result, for $\langle V A P\rangle$, reads

$$
\begin{aligned}
W_{\alpha \beta}(p, q)=\langle\bar{u} u\rangle\{ & \frac{\left(p_{\alpha}+2 q_{\alpha}\right) q_{\beta}-q^{2} g_{\alpha \beta}}{q^{2} l^{2}}+ \\
& \left.+\frac{F\left(p^{2}, q^{2}, l^{2}\right) P_{\alpha \beta}}{\left(p^{2}-M_{V}^{2}\right)\left(q^{2}-M_{A}^{2}\right) l^{2}}+\frac{G\left(p^{2}, q^{2}, l^{2}\right) Q_{\alpha \beta}}{\left(p^{2}-M_{V}^{2}\right)\left(q^{2}-M_{A}^{2}\right) q^{2} l^{2}}\right\}
\end{aligned}
$$

with

$$
\begin{aligned}
& F\left(p^{2}, q^{2}, l^{2}\right)=\frac{1}{2}\left(p^{2}-q^{2}-l^{2}\right)+M_{V}^{2}-M_{A}^{2} \\
& G\left(p^{2}, q^{2}, l^{2}\right)=-q^{2}+2 M_{A}^{2} .
\end{aligned}
$$

Let us compute the $V A P$ Green's function from the resonance lagrangian $\mathcal{L}_{\text {res }}^{(4)}+\mathcal{L}_{a_{1} \rho \pi}$. We find, for the $F$ and $G$ polynomials,

$$
\begin{aligned}
F\left(p^{2}, q^{2}, l^{2}\right)= & p^{2}\left[-\frac{F_{A}^{2}}{F_{0}^{2}}-\frac{F_{V} F_{A}}{F_{0}^{2}}\left(-2 G_{1}-G_{2}-2 G_{3}+H_{1}+H_{2}\right)\right]+ \\
& +q^{2}\left[\frac{F_{V}^{2}-2 G_{V} F_{V}}{F_{0}^{2}}-\frac{F_{V} F_{A}}{F_{0}^{2}}\left(G_{2}+2 G_{3}+H_{1}+H_{2}\right)\right]+ \\
& +l^{2}\left(\frac{-F_{V} F_{A}}{F_{0}^{2}}\right)\left[-G_{2}-2 G_{3}+H_{1}+H_{2}+4 H_{3}\right]+ \\
& +\frac{1}{F_{0}^{2}}\left[M_{V}^{2} F_{A}^{2}-M_{A}^{2}\left(F_{V}^{2}-2 G_{V} F_{V}\right)\right] \\
G\left(p^{2}, q^{2}, l^{2}\right)= & q^{2}\left[-\frac{2 G_{V} F_{V}}{F_{0}^{2}}-\frac{2 F_{V} F_{A}}{F_{0}^{2}}\left(-G_{1}+H_{1}+H_{2}\right)\right]+2 M_{A}^{2} \frac{G_{V} F_{V}}{F_{0}^{2}} .
\end{aligned}
$$

Let us first compare the constant terms in eqs. (5.11) and (5.10). Provided that the two Weinberg relations eq. (4.10) are satisfied together with the relation $G_{V} F_{V}=F_{0}^{2}$, one finds that the constant terms are in exact agreement. Next, the following three independent relations between the coupling constants $G_{i}, H_{i}$

$$
\begin{aligned}
G_{1}+G_{2}+2 G_{3} & =\frac{F_{A}}{F_{V}} \\
H_{1}+H_{2} & =\frac{F_{A}}{2 F_{V}}-\frac{F_{V}}{2 F_{A}}+G_{1} \\
2 H_{3} & =\frac{F_{V}}{2 F_{A}}-G_{1}
\end{aligned}
$$


are found to be the necessary and sufficient conditions for the polynomials in eqs. (5.11) and (5.10) to be completely identical.

\subsection{Linear contributions from $\mathcal{L}_{a_{1} \rho \pi}$}

Let us now return to the 4-point correlators and consider the contributions which are linear in the couplings of eq. (5.3). The couplings $H_{i}$ do not contribute because derivatives of the sources are involved. There are contributions to $\left\langle A A Q_{A} Q_{A}\right\rangle_{1},\left\langle A A Q_{V} Q_{V}\right\rangle_{1}$ and $\left\langle A V Q_{A} Q_{V}\right\rangle_{1}$ which we consider in turn. There is one kind of diagram relevant for $\left\langle A A Q_{A} Q_{A}\right\rangle_{1}$

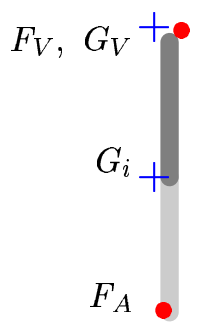

The upper vertex involves either $F_{V}$ or $G_{V}$. These diagrams give

$$
\begin{aligned}
\left\langle A A Q_{A} Q_{A}\right\rangle_{1}=-4 F_{A}\left(F_{V}-2 G_{V}\right)\left(1-\frac{1}{d}\right) \int \frac{-i d^{d} p}{(2 \pi)^{d}}[ & \frac{(d-2) G_{2}}{M_{V}^{2}\left(M_{A}^{2}-p^{2}\right)}+ \\
& \left.+\frac{G_{123}}{\left(M_{A}^{2}-p^{2}\right)\left(M_{V}^{2}-p^{2}\right)}\right]
\end{aligned}
$$

where

$$
G_{123} \equiv G_{1}+G_{2}+2 G_{3},
$$

denotes the combination which was determined from $\langle V A P\rangle$. In the case of the correlator $\left\langle A A Q_{V} Q_{V}\right\rangle_{1}$, there is again one kind of diagram which makes a linear contribution

$$
\begin{gathered}
F_{A} \\
G_{i}+ \\
F_{A}+。
\end{gathered}
$$

and the result is very similar to the preceding one

$$
\left\langle A A Q_{V} Q_{V}\right\rangle_{1}=-4 F_{V}^{2}\left(1-\frac{1}{d}\right) \int \frac{-i d^{d} p}{(2 \pi)^{d}}\left[\frac{(d-2) G_{1}}{M_{A}^{2}\left(M_{V}^{2}-p^{2}\right)}+\frac{G_{123}}{\left(M_{A}^{2}-p^{2}\right)\left(M_{V}^{2}-p^{2}\right)}\right] .
$$

Next, in order to obtain $\left\langle A V Q_{A} Q_{V}\right\rangle$ one must calculate a set of five diagrams which are depicted below, 

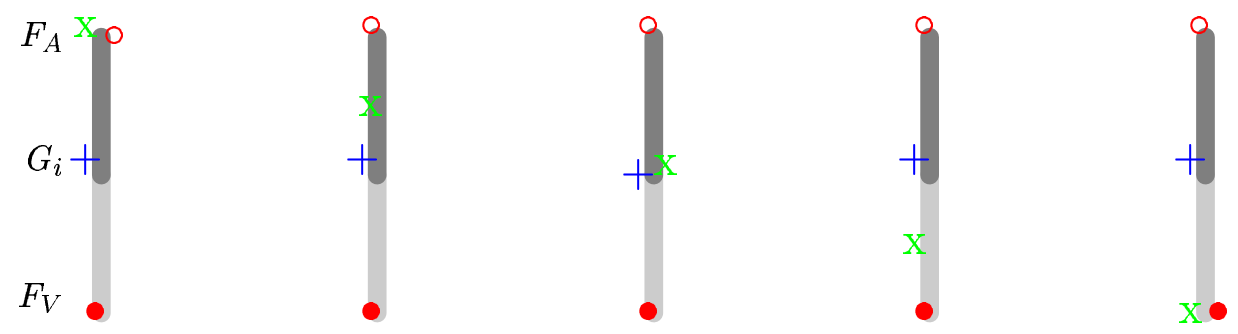

Computing these diagrams gives

$$
\begin{aligned}
\left\langle A V Q_{A} Q_{V}\right\rangle_{1}=-F_{A} F_{V} G_{123}\left(1-\frac{1}{d}\right) \int \frac{-i d^{d} p}{(2 \pi)^{d}}[ & \frac{d-2}{\left(M_{A}^{2}-p^{2}\right)\left(M_{V}^{2}-p^{2}\right)}+ \\
& +\frac{2 M_{A}^{2}}{\left(M_{A}^{2}-p^{2}\right)^{2}\left(M_{V}^{2}-p^{2}\right)}+ \\
& \left.+\frac{2 M_{V}^{2}}{\left(M_{A}^{2}-p^{2}\right)\left(M_{V}^{2}-p^{2}\right)^{2}}\right]
\end{aligned}
$$

which is seen to be proportional to the coupling constant combination $G_{123}$.

5.4 Quadratic contributions from $\mathcal{L}_{a_{1} \rho \pi}$ and consistency conditions for $\Pi_{1}, \Pi_{2}$, $\Pi_{8}$

We turn now to the contributions which are quadratic in the couplings $G_{i}$. These concern only the two correlators $\left\langle A A Q_{A} Q_{A}\right\rangle_{1},\left\langle A A Q_{V} Q_{V}\right\rangle_{1}$ and they are given respectively from the two kinds of diagrams depicted below

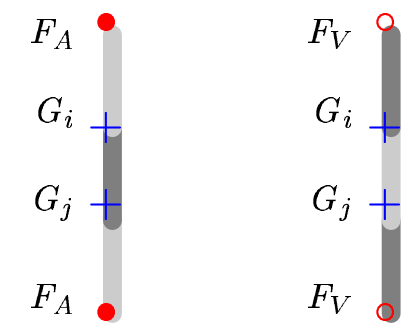

The computation gives the following result for $\left\langle A A Q_{A} Q_{A}\right\rangle_{1}$,

$$
\left\langle A A Q_{A} Q_{A}\right\rangle_{1}=4 F_{A}^{2}\left(1-\frac{1}{d}\right) \int \frac{-i d^{d} p}{(2 \pi)^{d}}\left\{\frac{\left(G_{123}\right)^{2} p^{2}}{\left(M_{A}^{2}-p^{2}\right)^{2}\left(M_{V}^{2}-p^{2}\right)}+\frac{G_{2}^{2}(2-d) p^{2}}{M_{V}^{2}\left(M_{A}^{2}-p^{2}\right)^{2}}\right\} .
$$

The result for $\left\langle A A Q_{V} Q_{V}\right\rangle_{1}$ is obtained simply by replacing $F_{A}$ by $F_{V}$ and by interchanging $M_{A}, M_{V}$ and $G_{1}, G_{2}$ in the expression above.

We can now collect all the contributions which involve the coupling constants $G_{i}$,

$$
\begin{aligned}
\left\langle A A Q_{A} Q_{A}\right\rangle_{1}^{G_{i}}=\frac{3 F_{A}\left(F_{V}-2 G_{V}\right)}{16 \pi^{2}}\{ & -2 G_{2} z\left(\frac{7}{6}+\log \frac{M_{A}^{2}}{\Lambda^{2}}\right)+ \\
& \left.+G_{123}\left[\frac{1}{6}+\frac{z \log z}{z-1}+\log \frac{M_{V}^{2}}{\Lambda^{2}}\right]\right\}+
\end{aligned}
$$




$$
\begin{aligned}
& +\frac{3 F_{A}^{2}}{16 \pi^{2}}\left\{4 G_{2}^{2} z\left(\frac{5}{3}+\log \frac{M_{A}^{2}}{\Lambda^{2}}\right)-\right. \\
& \left.-\left(G_{123}\right)^{2}\left[\frac{7 z-1}{6(z-1)}-\frac{\log z}{(z-1)^{2}}+\log \frac{M_{A}^{2}}{\Lambda^{2}}\right]\right\} \\
& \left\langle A A Q_{V} Q_{V}\right\rangle_{1}^{G_{i}}=\frac{3 F_{A} F_{V}}{16 \pi^{2}}\left\{\frac{-2 G_{1}}{z}\left(\frac{7}{6}+\log \frac{M_{V}^{2}}{\Lambda^{2}}\right)+\right. \\
& \left.+G_{123}\left[\frac{1}{6}+\frac{z \log z}{z-1}+\log \frac{M_{V}^{2}}{\Lambda^{2}}\right]\right\}+ \\
& +\frac{3 F_{V}^{2}}{16 \pi^{2}}\left\{4 \frac{G_{1}^{2}}{z}\left(\frac{5}{3}+\log \frac{M_{V}^{2}}{\Lambda^{2}}\right)-\right. \\
& \left.-\left(G_{123}\right)^{2}\left[\frac{7-z}{6(1-z)}+\frac{z^{2} \log z}{(z-1)^{2}}+\log \frac{M_{V}^{2}}{\Lambda^{2}}\right]\right\}, \\
& \left\langle A V Q_{A} Q_{V}\right\rangle_{1}^{G_{i}}=\frac{3}{2} \frac{F_{A} F_{V} G_{123}}{16 \pi^{2}}\left[\frac{1}{6}+\frac{z \log z}{z-1}+\log \frac{M_{V}^{2}}{\Lambda^{2}}\right] .
\end{aligned}
$$

Adding these contributions to those computed in section 3 it is now possible to satisfy the consistency conditions associated with $\Pi_{1}, \Pi_{2}$ and $\Pi_{8}$. Firstly one observes that the $\Lambda$ scale dependence drops out from the combination $\Pi_{8}$ automatically provided the first relation (5.12) holds for $G_{123}$. Requiring that the consistency conditions for $\Pi_{1}$ and $\Pi_{2}$ be satisfied yields two Weinberg-type relations for $G_{1}$ and $G_{2}$

$$
\begin{aligned}
8 G_{1}^{2}-\frac{4}{\sqrt{z}} G_{1}-1 & =0 \\
8 G_{2}^{2}-\frac{4}{\sqrt{z}}(2-z) G_{2}-1 & =0 .
\end{aligned}
$$

The previous determinations of $F_{V}, F_{A}, G_{V}$ and of the combination $G_{1}+G_{2}+2 G_{3}$ have been used. Since these equations are quadratic, one could be concerned about the existence of real solutions. Real solutions do exist for any value of the mass ratio $z$ and, a priori, one has a set of four different solutions. By studying the behaviour as a function of $z$ we can reduce the multiplicity. We note that the Weinberg equations (4.10) have real solutions provided $z$ lies in the range $1 \leq z<\infty$. Firstly, let $z \rightarrow 1$ : this corresponds to a situation where chiral symmetry gets restored and one must have $G_{1}=G_{2}$ in this case. This argument reduces the multiplicity to just two solutions, which we can write,

$$
\begin{aligned}
G_{1} & =\frac{1}{4 \sqrt{z}}(1+\sigma \sqrt{1+2 z}) \\
G_{2} & =\frac{1}{4 \sqrt{z}}\left(2-z+\sigma \sqrt{(2-z)^{2}+2 z}\right), \quad \sigma= \pm 1 .
\end{aligned}
$$

We can further reduce the multiplicity by observing the behaviour as $z \rightarrow \infty$. In this limit, $G_{1}$ remains finite while in the case of $G_{2}$ only one solution remains finite corresponding to $\sigma=1$. Correspondingly, the divergence in $\Pi_{1}$ is logarithmic for $\sigma=1$ while it is linear 
for $\sigma=-1$. The solution with $\sigma=1$, therefore, appears to be the most plausible choice. Using this solution, the following determinations are obtained for $\Pi_{1}, \Pi_{2}$ and $\Pi_{8}$

$$
\begin{aligned}
& \Pi_{1}=\frac{3}{2} \frac{F_{0}^{2}}{16 \pi^{2}}\{ \log \frac{M_{\gamma}^{2}}{M_{V}^{2}}+\frac{\left(z^{3}-5 z^{2}+5 z+1\right) \log z}{(z-1)^{3}}+ \\
&\left.+\frac{z^{3}-2 z^{2}+7 z-10}{2(z-1)^{2}}-\frac{(z-2) \sqrt{z^{2}-2 z+4}}{2(z-1)}\right\} \\
& \Pi_{2}=\frac{3}{2} \frac{F_{0}^{2}}{16 \pi^{2}}\left\{\log \frac{M_{\gamma}^{2}}{M_{V}^{2}}-\frac{2 z^{2} \log z}{(z-1)^{3}}+\right. \\
&\left.\quad+\frac{6 z^{3}-7 z^{2}+6 z-1}{2 z(z-1)^{2}}+\frac{\sqrt{2 z+1}}{2 z(z-1)}\right\} \\
& \Pi_{8}=\frac{3}{8} \frac{F_{0}^{2}}{16 \pi^{2}}\left(-\log \frac{M_{\gamma}^{2}}{M_{V}^{2}}+\frac{(3 z+1) \log z}{(z-1)^{2}}-\frac{4 z}{z-1}\right) .
\end{aligned}
$$

\subsection{Contributions from $\mathcal{L}_{a_{0} f_{1} \pi}$ and the consistency condition for $\Pi_{3}$}

By analogy with the preceding we now consider couplings which involve the scalar mesons of the type $a_{0} f_{1} \pi$ which will contribute to $\left\langle A A Q_{A} Q_{A}\right\rangle_{2,3}$. There is only one lagrangian term which matters, shown in eq. (5.4) (assuming nonet symmetry). The term in which the derivative acts on $u_{\nu}$ does not contribute to our correlators nor to the physical decay amplitude which is discussed below. There are two kinds of diagrams which contribute to the relevant correlators

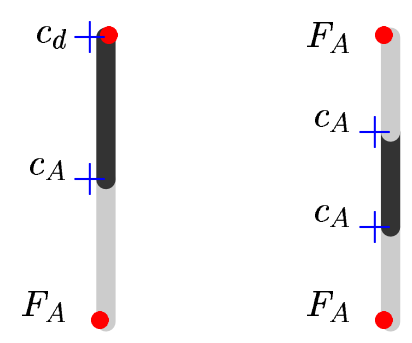

Computing the diagrams gives

$$
\begin{aligned}
\left\langle A A Q_{A} Q_{A}\right\rangle_{3}= & \left(1-\frac{1}{d}\right) \int \frac{-i d^{d} p}{(2 \pi)^{d}}\left\{\frac{8 \sqrt{2} c_{d} c_{A} F_{A}}{\left(M_{A}^{2}-p^{2}\right)\left(M_{S}^{2}-p^{2}\right)}+\frac{4 c_{A}^{2} F_{A}^{2} p^{2}}{\left(M_{A}^{2}-p^{2}\right)^{2}\left(M_{S}^{2}-p^{2}\right)}\right\} \\
= & \frac{-6 \sqrt{2} c_{A} c_{d} F_{A}}{16 \pi^{2}}\left(\frac{1}{6}+\log \frac{M_{S}^{2}}{\Lambda^{2}}+\frac{M_{A}^{2}}{M_{A}^{2}-M_{S}^{2}} \log \frac{M_{A}^{2}}{M_{S}^{2}}\right)+ \\
& +\frac{3 c_{A}^{2} F_{A}^{2}}{16 \pi^{2}}\left(\frac{1}{6}+\log \frac{M_{S}^{2}}{\Lambda^{2}}+\frac{M_{A}^{2}}{M_{A}^{2}-M_{S}^{2}}+\frac{M_{A}^{2}\left(M_{A}^{2}-2 M_{S}^{2}\right)}{\left(M_{A}^{2}-M_{S}^{2}\right)^{2}} \log \frac{M_{A}^{2}}{M_{S}^{2}}\right) .
\end{aligned}
$$

We can now add this result to the contribution (3.17) proportional to $c_{d}^{2}$, and the consistency condition translates into a simple relation between the coupling constants $c_{d}$ and $c_{a}$

$$
c_{A} F_{A}=\sqrt{2} c_{d} .
$$


This allows one to obtain a consistent determination of the correlator component $\left\langle A A Q_{A} Q_{A}\right\rangle_{3}$ associated with the scalar mesons,

$$
\Pi_{3}=\left\langle A A Q_{A} Q_{A}\right\rangle_{3}=\frac{6 c_{d}^{2} M_{A}^{2}}{16 \pi^{2}\left(M_{A}^{2}-M_{S}^{2}\right)}\left(1-\frac{M_{A}^{2}}{M_{A}^{2}-M_{S}^{2}} \log \frac{M_{A}^{2}}{M_{S}^{2}}\right) .
$$

We can compare the above determination of the coupling $c_{A}$ with experiment. Let us consider the decay process $f_{1}(1285) \rightarrow a_{0}(980)+\pi$. Using the lagrangian above (5.4) the expression for the decay width is easily derived,

$$
\Gamma_{f_{1} \rightarrow a_{0} \pi}=c_{A}^{2} \frac{p_{c m}\left(M_{A}^{2}-M_{S}^{2}\right)^{2}}{8 \pi M_{f_{1}}^{2} F_{0}^{2}} .
$$

Using the value $c_{d} \simeq 32 \mathrm{MeV}$ from ref. [35], $M_{A}=\sqrt{2} M_{\rho}$ (and physical masses in kinematical factors) one obtains

$$
\Gamma_{f_{1} \rightarrow a_{0} \pi} \simeq 7.7 \mathrm{MeV}
$$

This result is in rather good agreement with the experimental one $\Gamma_{f_{1} \rightarrow a_{0} \pi}^{\exp }=8.6 \pm 1.7 \mathrm{MeV}$ from ref. [4].

\subsection{Contributions from $\mathcal{L}_{\omega \rho \pi}$ in the vector vs. tensor formalism and $\Pi_{4}$}

We discuss here contributions from $\mathcal{L}_{\omega \rho \pi}$ eq. (5.5) which are likely to be important and were also not accounted for in the minimal resonance lagrangian. One faces a technical difficulty that the lagrangian eq. (5.5) involves the Levi-Civita $\epsilon$ tensor and this makes continuation to $d$ dimensional space ambiguous. One would therefore like to impose a stronger version of the consistency condition that the integral be finite in four dimensions. This condition is rather simple to enforce if one uses the vector formalism. Let us first briefly review what would be the result in this case and then we will show how to proceed in the case of the tensor formalism. An extensive study of resonance lagrangian terms in the vector formalism can be found in ref. [45]. In this formulation, the terms including the $\omega \rho \pi$ type couplings read (we use the same notation as [35]),

$$
\begin{aligned}
\mathcal{L}_{\omega \rho \pi}^{V}= & -\frac{1}{4}\left\langle V_{\mu \nu} V^{\mu \nu}-2 m_{V}^{2} V_{\mu} V^{\mu}\right\rangle-\frac{1}{2 \sqrt{2}} f_{V}\left\langle V_{\mu \nu} f_{+}^{\mu \nu}\right\rangle+ \\
& +\frac{g_{1}^{V}}{2 \sqrt{2}} \epsilon^{\mu \nu \alpha \beta}\left\langle\left\{u_{\mu}, V_{\nu}\right\} f_{+\alpha \beta}\right\rangle+\frac{g_{2}^{V}}{2} \epsilon^{\mu \nu \alpha \beta}\left\langle\left\{u_{\mu}, V_{\nu}\right\} V_{\alpha \beta}\right\rangle,
\end{aligned}
$$

where

$$
V_{\mu \nu}=\nabla_{\mu} V_{\nu}-\nabla_{\nu} V_{\mu}
$$

In this description the consideration of short distance conditions imposes that chiral $O\left(p^{4}\right)$ terms be added [35] but these do not play any role for our calculation. Computing the correlator $\left\langle A A Q_{V} Q_{V}\right\rangle_{3}$ from this lagrangian, one finds

$$
\left\langle A_{\alpha} A_{\beta} Q_{V} Q_{V}\right\rangle_{3}=8 \int \frac{d^{4} p}{(2 \pi)^{4}} \frac{\left(p^{2} g_{\alpha \beta}-p_{\alpha} p_{\beta}\right)\left[p^{2}\left(g_{1}^{V}+2 g_{2}^{V} f_{V}\right)-M_{V}^{2} g_{1}^{V}\right]^{2}}{p^{2}\left(M_{V}^{2}-p^{2}\right)^{3}}
$$


The integral in eq. (5.31) can be defined in four dimensions without difficulty, it suffices that the following relation holds among the coupling constants

$$
g_{1}^{V}+2 g_{2}^{V} f_{V}=0 \text {. }
$$

The result for the correlator then reads,

$$
\left\langle A A Q_{V} Q_{V}\right\rangle_{3}^{\omega \rho \pi}=\frac{F_{0}^{2}}{16 \pi^{2}}\left(3 \tilde{g}_{V}^{2}\right), \quad \tilde{g}_{V}=\frac{g_{1}^{V} M_{V}}{F_{0}} .
$$

The value of $\tilde{g}_{V}$ was estimated in ref. [7] from the $\omega \rightarrow \pi \gamma$ decay rate, $\left|\tilde{g}_{V}\right|=0.91 \pm 0.03$. Under the assumption of nonet symmetry (which is rather well satisfied experimentally for the vectors), one also has

$$
\left\langle A A Q_{V} Q_{V}\right\rangle_{2}^{\omega \rho \pi}=\frac{2}{3}\left\langle A A Q_{V} Q_{V}\right\rangle_{3}^{\omega \rho \pi} .
$$

From eqs. (5.33), (5.34) one can deduce an estimate, which is identical to the one performed in ref. [7], for the combination of $K_{i}$ 's which is involved in the radiative corrections to the process $\pi^{0} \rightarrow 2 \gamma$.

Let us now reconsider the problem using the antisymmetric tensor formulation, i.e. starting from the lagrangian of eq. (5.5). One has three kinds of diagrams to compute which are shown below
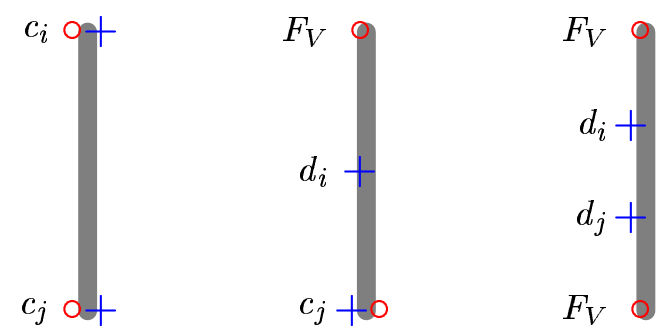

Evaluating these various diagrams we obtain $\left\langle A_{\alpha} A_{\beta} Q_{V} Q_{V}\right\rangle_{3}$ in the form of the following four-dimensional integral

$$
\begin{aligned}
\left\langle A_{\alpha} A_{\beta} Q_{V} Q_{V}\right\rangle_{3}=\int \frac{d^{4} p}{(2 \pi)^{4}}\{ & \frac{48 p_{\alpha} p_{\beta}}{M_{V}^{4}}\left(\frac{M_{V} F_{V}\left(d_{3}+d_{4}\right)}{M_{V}^{2}-p^{2}}-\sqrt{2}\left(c_{5}+c_{7}\right)\right)^{2}+ \\
& \left.+\frac{32\left(p^{2} g_{\alpha \beta}-p_{\alpha} p_{\beta}\right)}{M_{V}^{2}\left(M_{V}^{2}-p^{2}\right)}\left(\frac{2 M_{V} F_{V} d_{3}}{M_{V}^{2}-p^{2}}-\sqrt{2}\left(c_{5}-c_{6}\right)\right)^{2}\right\} .
\end{aligned}
$$

The integral in eq. (5.35) does not converge in four dimensions unless all the couplings $c_{i}$ and $d_{i}$ vanish identically. In order to remove the divergence, in this case, we introduce a more general set of resonance lagrangian terms, which include four building blocks

$$
\begin{aligned}
\mathcal{L}^{4 b b}= & \left(x_{1} g^{\mu_{0} \mu_{1}} g^{\mu_{2} \mu_{3}}+x_{2} g^{\mu_{0} \mu_{2}} g^{\mu_{1} \mu_{3}}+x_{3} g^{\mu_{0} \mu_{3}} g^{\mu_{1} \mu_{2}}\right)\left\langle\left\{f_{\mu_{0}}^{+\lambda}, u_{\mu_{1}}\right\}\left\{f_{\lambda \mu_{2}}^{+}, u_{\mu_{3}}\right\}\right\rangle+ \\
& +\sqrt{2}\left(y_{1} g^{\mu_{0} \mu_{1}} g^{\mu_{2} \mu_{3}}+y_{2} g^{\mu_{0} \mu_{2}} g^{\mu_{1} \mu_{3}}+y_{3} g^{\mu_{0} \mu_{3}} g^{\mu_{1} \mu_{2}}\right)\left\langle\left\{V_{\mu_{0}}^{\lambda}, u_{\mu_{1}}\right\}\left\{f_{\lambda \mu_{2}}^{+}, u_{\mu_{3}}\right\}\right\rangle+ \\
& +\left(z_{1} g^{\mu_{0} \mu_{1}} g^{\mu_{2} \mu_{3}}+z_{2} g^{\mu_{0} \mu_{2}} g^{\mu_{1} \mu_{3}}+z_{3} g^{\mu_{0} \mu_{3}} g^{\mu_{1} \mu_{2}}\right)\left\langle\left\{V_{\mu_{0}}^{\lambda}, u_{\mu_{1}}\right\}\left\{V_{\lambda \mu_{2}}, u_{\mu_{3}}\right\}\right\rangle .
\end{aligned}
$$


These terms generate the following additional contribution to the correlator

$$
\begin{aligned}
\left\langle A_{\alpha} A_{\beta} Q_{V} Q_{V}\right\rangle_{3}^{4 b b}=\int \frac{d^{4} p}{(2 \pi)^{4}}\{ & 24 p^{2} g_{\alpha \beta}\left(\frac{2 x_{123}}{p^{2}}-\frac{F_{V} y_{123}}{p^{2}\left(M_{V}^{2}-p^{2}\right)}+\frac{F_{V}^{2} z_{123}}{p^{2}\left(M_{V}^{2}-p^{2}\right)^{2}}\right)-(5.37) \\
& \left.-16\left(p^{2} g_{\alpha \beta}-p_{\alpha} p_{\beta}\right)\left(\frac{2 x_{13}}{p^{2}}-\frac{F_{V} y_{13}}{p^{2}\left(M_{V}^{2}-p^{2}\right)}+\frac{F_{V}^{2} z_{13}}{p^{2}\left(M_{V}^{2}-p^{2}\right)^{2}}\right)\right\}
\end{aligned}
$$

In this expression we have used the notation

$$
x_{123}=x_{1}+2 x_{2}+x_{3}, \quad x_{13}=x_{1}+x_{3},
$$

and similarly with $y_{i}, z_{i}$.

The convergence constraint in four dimension can now be satisfied: it imposes $c_{5}+c_{7}=$ 0 and determines the values of the six combinations of parameters which appear in eq. (5.37) in terms of $d_{3}, d_{3}+d_{4}$ and $c_{5}-c_{6}$. Collecting all the pieces, one finds that all the couplings drop out except $d_{3}$ and the correlator $\left\langle A_{\alpha} A_{\beta} Q_{V} Q_{V}\right\rangle_{3}$ is then expressed as a convergent integral,

$$
\left\langle A_{\alpha} A_{\beta} Q_{V} Q_{V}\right\rangle_{3}=128 M_{V}^{2} F_{V}^{2} d_{3}^{2} \int \frac{d^{4} p}{(2 \pi)^{4}} \frac{p^{2} g_{\alpha \beta}-p_{\alpha} p_{\beta}}{p^{2}\left(M_{V}^{2}-p^{2}\right)^{3}} .
$$

This expression is exactly analogous to the one obtained upon using the vector formalism and we write the final result in the same form,

$$
\Pi_{4}=\left\langle A A Q_{V} Q_{V}\right\rangle_{3}=\frac{F_{0}^{2}}{16 \pi^{2}}\left(3 \tilde{g}_{A T}^{2}\right), \quad \tilde{g}_{A T}=\frac{4 F_{V} d_{3}}{F_{0}} .
$$

The value of $d_{3}$ may be determined by enforcing asymptotic conditions concerning the $\langle V V P\rangle$ correlator. Not all conditions can be satisfied at the same time if one uses a minimal resonance model [42, 43, 46]. If one chooses to enforce the overall scaling behaviour one obtains [11]

$$
d_{3}^{\text {scaling }}=\frac{F_{0}^{2}}{8 M_{V}^{2}}-\frac{N_{c} M_{V}^{2}}{64 \pi^{2} F_{V}^{2}} .
$$

Numerically, this gives $\tilde{g}_{A T}=-0.58$ which is somewhat smaller in magnitude than $\tilde{g}_{V}$. Alternatively, one may choose instead to impose that the $\langle V V P\rangle$ correlator satisfies the VMD property, in which case one gets

$$
d_{3}^{V M D}=-\frac{N_{c} M_{V}^{2}}{64 \pi^{2} F_{V}^{2}} .
$$

If one uses this latter determination, one gets $\tilde{g}_{A T} \simeq-0.93$, i.e. the result from the antisymmetric tensor formalism becomes essentially identical to the one from the vector formalism.

\section{Numerical results}

\subsection{Results for $K_{1}, \ldots, K_{6}$}

In the numerical applications we will simply use $M_{V}=0.77 \mathrm{GeV}$ and $F_{V}=\sqrt{2} F_{\pi}$ (KSRF relation 44]), which corresponds to $z=2$ (the same values were used by BU). We will also 


\begin{tabular}{|c|c|c|c|c|c|l|}
\hline $10^{3} K_{1}^{r}$ & $10^{3} K_{2}^{r}$ & $10^{3} K_{3}^{r}$ & $10^{3} K_{4}^{r}$ & $10^{3} K_{5}^{r}$ & $10^{3} K_{6}^{r}$ & \\
\hline-4.44 & -1.73 & 4.44 & -3.46 & 13.31 & 5.19 & $\rho, a_{1}$ \\
-6.8 & -2.7 & 6.8 & -5.5 & 20.3 & 8.2 & $\rho, a_{1}(\mathrm{BU})$ \\
\hline 1.97 & 1.97 & -1.97 & 3.93 & -1.97 & -1.97 & $\omega \rho \pi$ \\
\hline-0.35 & 0.35 & 0.35 & 0.69 & 0.35 & -0.35 & scalars \\
0.4 & -0.4 & -0.4 & -0.7 & -0.4 & 0.4 & scalars (BU) \\
\hline 0.11 & 0.11 & -0.11 & 0.21 & -0.11 & -0.11 & $b_{1}(1235)$ \\
\hline-2.71 & 0.69 & 2.71 & 1.38 & 11.59 & 2.77 & Total \\
\hline
\end{tabular}

Table 1: Numerical results obtained for $K_{i}^{r}(\mu)$ with $\mu=0.77 \mathrm{GeV}$ from the present work. The contributions associated with the $b_{1}(1235)$ are discussed in appendix B. The results from ref. [29] are also shown for comparison.

use $F_{0}=F_{\pi}=92.4 \mathrm{MeV}$. The results for $K_{1}^{r}(\mu), \ldots, K_{6}^{r}(\mu)$ with $\mu=M_{V}$ are collected in table 1 . We show separately the contributions due to the scalars (terms proportional to $c_{d}$, with $c_{d}=32 \mathrm{MeV}$ ) and the contributions from the $\omega \rho \pi$ interactions (terms proportional to $\tilde{g}_{A T}$, with $\left.\left|\tilde{g}_{A T}\right|=0.93\right)$. We find that this contribution can be significant for some of the couplings like $K_{2}^{r}$ or $K_{4}^{r}$. The contributions related to the scalars are rather small. In that case, our results agree on the magnitude but have a different sign from those of BU because of the extra contribution needed to ensure consistency. The largest contributions are those originating from the $a_{1} \rho \pi$ sector. In that case our numerical results are in qualitative agreement with those of $\mathrm{BU}$. We have also performed an estimate of the contributions associated with the $b_{1}$ meson (see appendix B) which turn out to be rather small.

\subsection{An update on the corrections to Dashen's theorem}

A reliable evaluation of the electromagnetic part of the $K^{+}-K^{0}$ mass difference is important for the determination of $m_{u}-m_{d}$. At leading order in the chiral expansion it is given by Dashen's theorem [26]

$$
\left(\Delta M_{K}^{2}\right)_{E M}=\left(\Delta M_{\pi}^{2}\right)_{E M}+O\left(e^{2} m_{q}\right) .
$$

It was pointed out that the corrections of order $e^{2} m_{s}$ could be rather large 48 such that the right hand side could be modified by as much as a factor of two. Using Urech's formalism one can compute explicitly these corrections which have the following form [四]

$$
\left(\Delta M_{K}^{2}\right)_{E M}-\left(\Delta M_{\pi}^{2}\right)_{E M}=e^{2} M_{K}^{2}\left(A_{1}+A_{2}+A_{3}\right)+O\left(e^{2} M_{\pi}^{2}\right)
$$

with

$$
\begin{aligned}
& A_{1}=-\frac{1}{16 \pi^{2}}\left[(3+2 Z) \log \frac{M_{K}^{2}}{\mu^{2}}-4\right]-16 Z L_{5}^{r}, \quad Z=\frac{C}{F_{0}^{4}} \\
& A_{2}=-\frac{4}{3}\left(K_{5}^{r}+K_{6}^{r}\right) \\
& A_{3}=8\left(K_{10}^{r}+K_{11}^{r}\right) .
\end{aligned}
$$

As discussed in (I) (and also in [30]) the Urech couplings $K_{10}^{r}, K_{11}^{r}$ taken separately suffer from short distance ambiguities and depend on the gauge. The sum, however, is perfectly 
well defined and can be estimated from sum rules related to the correlators $\left\langle V^{3} V^{3}-V^{8} V^{8}-\right.$ $\left.A^{3} A^{3}+A^{8} A^{8}\right\rangle$ and $\langle V A P\rangle$, giving the following result [31]

$$
\begin{aligned}
K_{10}^{r}+K_{11}^{r}=\frac{3}{16} \frac{1}{16 \pi^{2}}\{ & \log \frac{M_{V}^{2}}{\mu^{2}}+Z_{A}-Z_{V}+ \\
& \left.+2 \log z\left[\frac{(z+1)^{2}}{2(z-1)^{3}}+1\right]+\frac{2 z(z-3)}{(z-1)^{2}}+\frac{1}{6}+4 Z_{\mu}^{0}\right\}
\end{aligned}
$$

with

$$
Z_{A}-Z_{V}=\frac{4 M_{A}^{2}\left(F_{\pi}-F_{K}\right)}{F_{\pi}\left(M_{\pi}^{2}-M_{K}^{2}\right)}+\frac{2 F_{V}^{2} M_{\rho}\left(M_{\rho}-M_{\phi}\right)}{F_{\pi}^{2}\left(M_{\pi}^{2}-M_{K}^{2}\right)} \log z-2 \log z-2 .
$$

The quantity $Z_{A}-Z_{V}$ is generated from flavour symmetry breaking at first order in the vector multiplet as well as the axial-vector multiplet and the computation takes into account Weinberg-type sum rule relations for the splittings of the masses as well as the coupling constants. The last entry in the expression (6.4), $Z_{\mu}^{0}$, is a contribution which should restore the scale dependence proportional to $C / F_{0}^{4}$. Since this effect is subleading in $N_{c}$, this term will be dropped here for consistency with previous approximations. Making use of the result eq. (6.4) and of the numerical values for $K_{5}^{r}+K_{6}^{r}$ shown in table 1 we find the following numerical values for the three corrective terms (which we give in units of the physical $\pi^{+}-\pi^{0}$ mass difference, $\Delta M_{\pi}^{2}=1261.16 \mathrm{MeV}^{2}$ )

$$
\left(\Delta M_{K}^{2}\right)_{E M}-\left(\Delta M_{\pi}^{2}\right)_{E M}=\Delta M_{\pi}^{2}[0.59-0.34+1.25]
$$

The new result with respect to (I) concerns $K_{5}+K_{6}$ and we find that this contribution goes in the sense of reducing the size of the correction. This is in qualitative agreement with the result of $\mathrm{BU}$. However, the effect of the extra terms from $\mathcal{L}_{a_{1} \rho \pi}$ included here is to reduce the magnitude of $K_{5}+K_{6}$ by approximately a factor of two as compared to BU. There is a clear indication for a sizable correction to Dashen's theorem rather similar to that found in refs. [49, 30]. The origin of the different result obtained in ref. [29] can be traced, essentially, to the fact that their result for $K_{10}+K_{11}$ is ten times smaller than ours. This is due to two reasons 1 ) flavour symmetry breaking in the vector and the axial-vector multiplets was ignored in 29] such that $K_{10}$ was just set to zero and 2) the effect of the couplings from $\mathcal{L}_{a_{1} \rho \pi}$ which they did not take into account is to strongly enhance the value of $K_{11}$.

\subsection{Uncertainties}

One should keep in mind that there are uncertainties which are difficult to estimate quantitatively. These have two main sources. The first one is that we have used, at several places, large $N_{c}$ approximations a) in the treatment of the resonances, which are taken to be infinitely narrow and b) in the calculation of QCD n-point correlators in which only tree graphs were considered. The second source of uncertainty is that we have retained the contributions from the lightest resonances only. The usual experience with such approximations is that reasonable order of magnitudes should be obtained. 


\section{Conclusions}

In this paper, we have considered a set of sum rules for the electromagnetic chiral parameters which involve QCD 4-point correlators in connection with the construction of a chiral lagrangian with resonances. For our purposes, the resonance lagrangian provides rational-type approximations for the QCD correlators obeying the chiral Ward identities.

We have shown that the resonance lagrangian of order $p^{4}$ was not sufficient to ensure all the convergence conditions required by the sum rules. We have then shown that introducing a set of terms of order $p^{6}$ involving $a_{1} \rho \pi$-type couplings renders it possible to satisfy these conditions in the sense of finiteness as a $d \rightarrow 4$ limit. The new coupling constants which appear were shown to obey non linear equations, we have argued that physical requirements select a unique solution.

Our investigation, however, was essentially limited to a set of terms with three building blocks. A more general investigation should be performed in the future. A more complete determination of the resonance lagrangian would lead to much better estimates of the chiral coupling constants at this order [50] than those available at present. This would improve significantly the effectiveness of the chiral calculations at order $p^{6}$. There is also a limitation in the set of resonances which were included. In this regard, the role of tensor mesons should perhaps be investigated [01].

We have considered an application to the problem of the chiral corrections to Dashen's low-energy theorem. We have found that the combination $K_{5}^{r}+K_{6}^{r}$ as determined here reduces the size of the correction which still remains rather large. One motivation for deriving estimates for each of the parameters $K_{i}$ is to improve the accuracy of radiative correction evaluations. In this context, further work is still needed because another set of parameters, $X_{i}$, is involved in the interesting case of the semi-leptonic decays [5]. Finally, a formal similarity can be noted between the sum rules considered here and those shown recently to hold for chiral parameters in the weak non-leptonic sector [52].

\section{Acknowledgments}

This work is supported in part by IFCPAR contract 2504-1, by the European Union TMR network, Contract No. HPRN-CT-2002-00311 (EURIDICE) and by the Department of Science and Technology, Government of India.

\section{A. Vertices}

We list below the set of vertices which are needed in section 2 and those needed section 3 which are associated with vector and axial-vector resonances. The vertices represent derivatives of the action (times $i$ ) with respect to the various sources (with each source multiplied by $i$ ). It is not difficult to derive, in an analogous way, all the other relevant vertices. 


\section{A.1 Vertices with pions}

$$
V=-F \partial_{\lambda} \pi^{c}(z) F^{\lambda}(z)
$$

$$
V=i F f^{c a t} \pi^{t}(x) F_{\alpha}(x) \delta(x-z)
$$

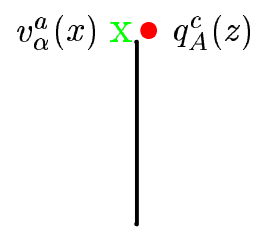

$$
V=i F f^{b c t} \pi^{t}(y) F_{\beta}(y) \delta(y-z)
$$

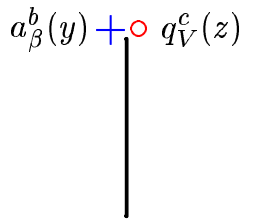

$$
V=i F f^{b a t} \pi^{t}(x) g^{\alpha \beta} \delta(x-y)
$$

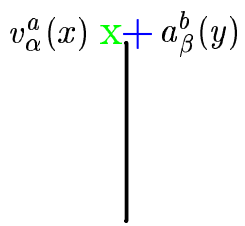

$$
V=-f^{s t c} \partial_{\mu} \pi^{s}(z) \pi^{t}(z) F_{\mu}(z)
$$

$$
V=-f^{s t a} \partial_{\alpha} \pi^{s}(x) \pi^{t}(x)
$$

$V=i g^{\alpha \beta} f^{a i s} f^{b j s} \pi^{i}(x) \pi^{j}(x) \delta(x-y)$

$a_{\alpha}^{a}(x)++a_{\beta}^{b}(y)$

$V=-i g^{\alpha \beta} f^{a i s} f^{b j s} \pi^{i}(x) \pi^{j}(x) \delta(x-y)$

$v_{\alpha}^{a}(x) \quad \times \mid v_{\beta}^{b}(y)$ 


\section{A.2 Resonance vertices with $v_{\mu}$ sources}

These vertices are the same for vector or axial-vector mesons:

$$
\begin{array}{lr}
V=-f^{a i j} R_{\alpha \mu}^{i}(x) \partial^{\lambda} R_{\lambda}^{j \mu}(x) & \times v_{\alpha}^{a}(x) \\
V=i f^{a i l} f^{b j l} R_{\alpha \mu}^{i}(x) R_{\beta}^{j \mu}(x) \delta(x-y) & v_{\alpha}^{a}(x) \quad \mathrm{XX} v_{\beta}^{b}(y)
\end{array}
$$

A.3 Vertices proportional to $F_{A}$

$$
\begin{aligned}
& V=-F_{A} A_{\mu \nu}^{c}(z) \partial^{\mu} F^{\nu}(z) \\
& V=i F_{A} f^{i b c} A_{\beta \lambda}^{i}(y) F^{\lambda}(y) \delta(y-z) \\
& V=i F_{A} f^{i a d} A_{\alpha \lambda}^{i}(x) F^{\lambda}(x) \delta(x) \\
& v_{\beta}^{b}(y) \quad \times q_{A}^{c}(z) \\
& V=\frac{-F_{A}}{F} f^{d i j} A_{\mu \nu}^{i}(0) \partial^{\mu} F^{\nu}(0) \pi^{j}(0) \\
& a_{\alpha}^{a}(x)+\circ q_{V}^{d}(0) \\
& V=\frac{F_{A}}{F} f^{i j l} f^{a b l} A_{\alpha \beta}^{i}(x) \pi^{j}(x) \delta(x-y) \\
& v_{\alpha}^{a}(x) \quad{ }_{\mathrm{x}}^{d}(0)
\end{aligned}
$$

Note that this vertex is antisymmetric in the Lorentz indices $\alpha \beta$ and will therefore not contribute here. 
A.4 Vertices proportional to $F_{V}$

$$
\begin{aligned}
& V=F_{V} V_{\mu \nu}^{c}(z) \partial^{\mu} F^{\nu}(z) \\
& v_{\alpha}^{a}(x) \quad \times \circ q_{V}^{c}(z) \\
& V=-i F_{V} f^{i a c} V_{\alpha \lambda}^{i}(x) F^{\lambda}(x) \delta(x-z) \\
& V=-i F_{V} f^{i a c} V_{\alpha \lambda}^{i}(x) F^{\lambda}(x) \delta(x-z) \\
& V=\frac{F_{V}}{F} f^{s t c} V_{\mu \nu}^{s} \partial^{\mu} F^{\nu} \pi^{t} \\
&
\end{aligned}
$$

\section{A.5 Vertices proportional to $G_{V}$}

$$
a_{\alpha}^{a}(x)+\bullet q_{A}^{c}(z)
$$

$$
V=2 i G_{V} f^{a c t} V_{\alpha \nu}^{t}(x) F^{\nu}(x) \delta(x-z)
$$

$$
V=\frac{-2 G_{V}}{F} f^{a s t} V_{\alpha \mu}^{s}(x) \partial^{\mu} \pi^{t}(x)
$$

$$
a_{\alpha}^{a}(x)+
$$

$$
V=\frac{-2 G_{V}}{F} f^{c s t} V_{\lambda \mu}^{s}(z) \partial^{\mu} \pi^{t}(z) F^{\lambda}(z)
$$

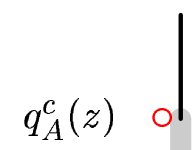

$$
V=\frac{-i G_{V}}{F_{0}^{2}} f^{s t u} \int d v V_{\mu \nu}^{s}(v) \partial^{\mu} \pi^{t}(v) \partial^{\nu} \pi^{u}(v)
$$

There are other potentially relevant vertices which are antisymmetric in $\alpha \beta$ and will thus not contribute for our purposes. 


\section{B. $b_{1}(1235)$ contributions}

We present here an estimate of the contributions associated with the $b_{1}(1235)$ resonance. The physics of the $b_{1}$ meson is not as constrained as that of the $a_{1}$ meson by chiral symmetry, so in this section we will occasionally make some educated guesses. The order of magnitude of the $b_{1}$ effect should nevertheless be properly accounted for. The contributions are to the same correlator components as those from the $\omega \rho \pi$ couplings. In principle, the convergence constraints should be applied to the sum. We will apply,however, these constraints separately to the $b_{1}$ for the purpose of obtaining an order of magnitude estimate.

We can write down a set of vertices in exact analogy with the $a_{1} \rho \pi$ vertices

$$
\begin{aligned}
\mathcal{L}_{b_{1} \omega \pi}= & \left(G_{1}^{B} g^{\mu_{0} \mu_{1}} g^{\mu_{2} \mu 3}+G_{2}^{B} g^{\mu_{0} \mu_{2}} g^{\mu_{3} \mu_{1}}+G_{3}^{B} g^{\mu_{0} \mu_{3}} g^{\mu_{1} \mu_{2}}\right)\left\langle\left\{\nabla_{\mu_{0}} V_{\mu_{1} \lambda}, B_{\mu_{2}}{ }^{\lambda}\right\} u_{\mu_{3}}\right\rangle+ \\
& +\left(H_{1}^{B} g^{\mu_{0} \mu_{1}} g^{\mu_{2} \mu_{3}}+H_{2}^{B} g^{\mu_{0} \mu_{2}} g^{\mu_{3} \mu_{1}}+H_{3}^{B} g^{\mu_{0} \mu_{3}} g^{\mu_{1} \mu_{2}}\right)\left\langle\left\{V_{\mu_{1} \lambda}, B_{\mu_{2}}{ }^{\lambda}\right\} \nabla_{\mu_{0}} u_{\mu_{3}}\right\rangle .
\end{aligned}
$$

The only difference with the lagrangian of eq. (5.3) is that the commutator is replaced by an anticommutator on account of the fact that the $b_{1}$ meson is odd under charge conjugation. As a consequence, the contribution to the correlator $\left\langle A A Q_{V} Q_{V}\right\rangle_{3}$ can be deduced simply from the results of section 5.4

$$
\begin{gathered}
\left\langle A A Q_{V} Q_{V}\right\rangle_{3}^{b_{1} \omega \pi}=\frac{-3 F_{V}^{2}}{16 \pi^{2}}\left\{-\left(G_{1}^{B}+G_{2}^{B}+2 G_{3}^{B}\right)^{2}\left[\log \frac{M_{V}^{2}}{\Lambda^{2}}+\frac{M_{B}^{4} \log \frac{M_{B}^{2}}{M_{V}^{2}}}{\left(M_{B}^{2}-M_{V}^{2}\right)^{2}}+\frac{7 M_{V}^{2}-M_{B}^{2}}{6\left(M_{V}^{2}-M_{B}^{2}\right)}\right]+\right. \\
\left.+4 \frac{\left(G_{1}^{B}\right)^{2} M_{V}^{2}}{M_{B}^{2}}\left(\log \frac{M_{V}^{2}}{\Lambda^{2}}+\frac{5}{3}\right)\right\} .
\end{gathered}
$$

Imposing a consistency condition on eq. (B.2) enables one to express $\left\langle A A Q_{V} Q_{V}\right\rangle_{3}^{b_{1} \omega \pi}$ in terms of the combination

$$
\tilde{G}^{B} \equiv G_{1}^{B}+G_{2}^{B}+2 G_{3}^{B}
$$

and one obtains

$$
\left\langle A A Q_{V} Q_{V}\right\rangle_{3}^{b_{1} \omega \pi}=\frac{3 F_{V}^{2}}{16 \pi^{2}}\left(\tilde{G}^{B}\right)^{2}\left[\frac{M_{B}^{4} \log \frac{M_{B}^{2}}{M_{V}^{2}}}{\left(M_{B}^{2}-M_{V}^{2}\right)^{2}}+\frac{3 M_{B}^{2}+M_{V}^{2}}{2\left(M_{B}^{2}-M_{V}^{2}\right)}\right] .
$$

We must next try to determine the relevant combination of couplings $\tilde{G}^{B}$ from experiment. The widths of the decays $b_{1}^{+} \rightarrow \gamma \pi^{+}$and $b_{1}^{+} \rightarrow \omega \pi^{+}$are known

$$
\Gamma_{b_{1}^{+} \rightarrow \gamma \pi^{+}}=227 \pm 59 \mathrm{KeV}, \quad \Gamma_{b_{1}^{+} \rightarrow \omega \pi^{+}}=142 \pm 9 \mathrm{MeV}
$$

and their expressions in terms of the resonance lagrangian coupling constants read

$$
\begin{aligned}
\Gamma_{b_{1}^{+} \rightarrow \gamma \pi^{+}} & =\frac{\alpha\left(M_{B}^{2}-M_{\pi}^{2}\right)}{24 M_{B}}\left(\frac{F_{V} M_{B}^{2}}{3 F_{\pi} M_{V}^{2}}\right)^{2}\left(\tilde{H}^{B}\right)^{2} \\
\Gamma_{b_{1}^{+} \rightarrow \omega \pi^{+}} & =\frac{p_{c m}\left(M_{B}^{2}-M_{\omega}^{2}\right)^{2}}{48 \pi M_{B}^{4} M_{\omega}^{2} F_{\pi}^{2}}\left\{2 M_{B}^{2} M_{V}^{2}\left(\tilde{G}^{B}\right)^{2}+\left[\left(M_{B}^{2}-M_{V}^{2}\right) \tilde{H}^{B}+2 M_{V}^{2} \tilde{G}^{B}\right]^{2}\right\} .
\end{aligned}
$$


Here, we have introduced the notation

$$
\tilde{H}^{B} \equiv H_{1}^{B}+H_{2}^{B}+G_{2}^{B}+2 G_{3}^{B} .
$$

Using these two pieces of information enables one to determine the combination of couplings that one needs, but there are several solutions due to the non linearity of the equations

$$
\tilde{H}^{B}= \pm 0.68, \quad \tilde{G}^{B} \equiv G_{1}^{B}+G_{2}^{B}+2 G_{3}^{B}= \pm 0.24, \pm 0.67 .
$$

We can eliminate some of the solutions by using one more piece of experimental information concerning the $D / S$ ratio in the decay $b_{1} \rightarrow \omega \pi$

$$
\frac{f_{D}}{f_{S}}=0.29 \pm 0.04
$$

The $S$ and the $D$-wave components of the decay amplitude $b_{1} \rightarrow \omega \pi$ (defined as in ref. [53]) are easily computed from our lagrangian

$$
\begin{aligned}
& f_{S}=-\frac{\sqrt{4 \pi}}{3 M_{B} M_{\omega} F_{\pi}}\left(M_{B}^{2}-M_{V}^{2}\right)\left[M_{V}\left(M_{B}+2 M_{V}\right) \tilde{G}^{B}+\left(M_{B}^{2}-M_{V}^{2}\right) \tilde{H}^{B}\right] \\
& f_{D}=-\frac{\sqrt{8 \pi}}{6 M_{B} M_{\omega} F_{\pi}}\left(M_{B}^{2}-M_{V}^{2}\right)\left[2 M_{V}\left(M_{B}-M_{V}\right) \tilde{G}^{B}+\left(M_{B}^{2}-M_{V}^{2}\right) \tilde{H}^{B}\right] .
\end{aligned}
$$

Using this result and the experimental one on the $D / S$ ratio reduces the set of acceptable solutions for the coupling constants to

$$
\left(\tilde{G}^{B}, \tilde{H}^{B}\right)= \pm(0.24,0.68)
$$

which give $f_{D} / f_{S}=0.28$.

\section{References}

[1] S. Weinberg, Phenomenological lagrangians, Physica 96 (1979) 327.

[2] J. Gasser and H. Leutwyler, Chiral perturbation theory to one loop, Ann. Phys. (NY) 158 (1984) 142 .

[3] J. Gasser and H. Leutwyler, Chiral perturbation theory: expansions in the mass of the strange quark, Nucl. Phys. B 250 (1985) 465.

[4] R. Urech, Virtual photons in chiral perturbation theory, Nucl. Phys. B 433 (1995) 234 hep-ph/9405341.

[5] M. Knecht, H. Neufeld, H. Rupertsberger and P. Talavera, Chiral perturbation theory with virtual photons and leptons, Eur. Phys. J. C 12 (2000) 469 hep-ph/9909284.

[6] G. Ecker, G. Isidori, G. Muller, H. Neufeld and A. Pich, Electromagnetism in nonleptonic weak interactions, Nucl. Phys. B 591 (2000) 419 hep-ph/0006172.

[7] B. Ananthanarayan and B. Moussallam, Electromagnetic corrections in the anomaly sector, J. High Energy Phys. 05 (2002) 052 hep-ph/0205232.

[8] U.G. Meissner and S. Steininger, Isospin violation in pion nucleon scattering, Phys. Lett. B 419 (1998) 403 hep-ph/9709453. 
[9] U.-G. Meissner, G. Muller and S. Steininger, Virtual photons in SU(2) chiral perturbation theory and electromagnetic corrections to $\pi \pi$ scattering, Phys. Lett. B 406 (1997) 154 hep-ph/9704377, erratum ibid. B 407 (1997) 454.

[10] M. Knecht and R. Urech, Virtual photons in low energy $\pi \pi$ scattering, Nucl. Phys. B 519 (1998) 329 hep-ph/9709348.

[11] B. Kubis and U.-G. Meissner, Isospin violation in pion kaon scattering, Nucl. Phys. A 699 (2002) 709 hep-ph/0107199.

[12] B. Kubis and U.-G. Meissner, Isospin violation in low-energy charged pion kaon scattering, Phys. Lett. B 529 (2002) 69 hep-ph/0112154.

[13] A. Nehme and P. Talavera, Isospin breaking corrections to low-energy $\pi-K$ scattering, Phys. Rev. D 65 (2002) 054023 hep-ph/0107299.

[14] M. Knecht and A. Nehme, Electromagnetic corrections to charged pion scattering at low energies, Phys. Lett. B 532 (2002) 55 hep-ph/0201033.

[15] A. Nehme, Isospin breaking in low-energy charged pion and kaon elastic scattering, Eur. Phys. J. C 23 (2002) 707 hep-ph/0111212.

[16] H. Sazdjian, The pionium lifetime in generalized chiral perturbation theory, Phys. Lett. B $490(2000) 203$ hep-ph/0004226.

[17] J. Gasser, V.E. Lyubovitskij, A. Rusetsky and A. Gall, Decays of the $\pi^{+} \pi^{-}$atom, Phys. Rev. D 64 (2001) 016008 hep-ph/0103157.

[18] J. Schweizer, Decay widths and energy shifts of $\pi \pi$ and $\pi K$ atoms, Phys. Lett. B 587 (2004) 33 hep-ph/0401048.

[19] V. Cirigliano, M. Knecht, H. Neufeld, H. Rupertsberger and P. Talavera, Radiative corrections to $K_{\ell 3}$ decays, Eur. Phys. J. C 23 (2002) 121 hep-ph/0110153.

[20] A. Nehme, Isospin breaking in $K_{\ell 4}$ decays of the neutral kaon, Nucl. Phys. B 682 (2004) 289 hep-ph/0311113;

V. Cuplov and A. Nehme, Isospin breaking in $K_{\ell 4}$ decays of the charged kaon, hep-ph/0311274.

[21] L. Ametller, M. Knecht and P. Talavera, Electromagnetic corrections to gamma $\pi^{ \pm} \rightarrow \pi^{0} \pi^{ \pm}$, Phys. Rev. D 64 (2001) 094009 hep-ph/0107127.

[22] V. Cirigliano, G. Ecker and H. Neufeld, Isospin violation and the magnetic moment of the muon, Phys. Lett. B 513 (2001) 361 hep-ph/0104267.

[23] V. Cirigliano, J.F. Donoghue and E. Golowich, Electromagnetic corrections to $k \rightarrow$ pi pi. $i$ : chiral perturbation theory, Phys. Rev. D 61 (2000) 093001 hep-ph/9907341, erratum ibid. D 63 (2001) 059903.

[24] V. Cirigliano, G. Ecker, H. Neufeld and A. Pich, Isospin breaking in $K \rightarrow \pi \pi$ decays, Eur. Phys. J. C 33 (2004) 369 hep-ph/0310351.

[25] V. Cirigliano, A. Pich, G. Ecker and H. Neufeld, Isospin violation in $\epsilon^{\prime}$, Phys. Rev. Lett. 91 (2003) 162001 hep-ph/0307030.

[26] R.F. Dashen, Chiral $\mathrm{SU}(3) \times \mathrm{SU}(3)$ as a symmetry of the strong interactions, Phys. Rev. 183 (1969) 1245 . 
[27] H. Leutwyler, The ratios of the light quark masses, Phys. Lett. B 378 (1996) 313 hep-ph/9602366.

[28] J. Gasser, A. Rusetsky and I. Scimemi, Electromagnetic corrections in hadronic processes, Eur. Phys. J. C 32 (2003) 97 hep-ph/0305260.

[29] R. Baur and R. Urech, Resonance contributions to the electromagnetic low energy constants of chiral perturbation theory, Nucl. Phys. B 499 (1997) 319 hep-ph/9612328.

[30] J. Bijnens and J. Prades, Electromagnetic corrections for pions and kaons: masses and polarizabilities, Nucl. Phys. B 490 (1997) 239 hep-ph/9610360.

[31] B. Moussallam, A sum rule approach to the violation of Dashen's theorem, Nucl. Phys. B 504 (1997) 381 hep-ph/9701400.

[32] T. Das, G.S. Guralnik, V.S. Mathur, F.E. Low and J.E. Young, Electromagnetic mass difference of pions, Phys. Rev. Lett. 18 (1967) 759.

[33] G. 't Hooft, A planar diagram theory for strong interactions, Nucl. Phys. B 72 (1974) 461.

[34] G. Ecker, J. Gasser, A. Pich and E. de Rafael, The role of resonances in chiral perturbation theory, Nucl. Phys. B 321 (1989) 311.

[35] G. Ecker, J. Gasser, H. Leutwyler, A. Pich and E. de Rafael, Chiral lagrangians for massive spin 1 fields, Phys. Lett. B 223 (1989) 425.

[36] R. Baur and R. Urech, On the corrections to Dashen's theorem, Phys. Rev. D 53 (1996) 6552 hep-ph/9508393.

[37] H. Neufeld and H. Rupertsberger, Isospin breaking in chiral perturbation theory and the decays $\eta \rightarrow \pi$ lepton neutrino and $\tau \rightarrow \eta \pi$ neutrino, Z. Physik C 68 (1995) 91; The electromagnetic interaction in chiral perturbation theory, Z. Physik C $\mathbf{7 1}$ (1996) 131 hep-ph/9506448.

[38] E. de Rafael, Analytic approaches to kaon physics, Nucl. Phys. 119 (Proc. Suppl.) (2003) 71 hep-ph/0210317.

[39] D.J. Gross and R. Jackiw, Effect of anomalies on quasirenormalizable theories, Phys. Rev. D 6 (1972) 477 .

[40] V. Cirigliano, G. Ecker, M. Eidemuller, A. Pich and J. Portoles, The $\langle V A P\rangle$ Green function in the resonance region, hep-ph/0404004.

[41] P.D. Ruiz-Femenia, A. Pich and J. Portoles, Odd-intrinsic-parity processes within the resonance effective theory of QCD, J. High Energy Phys. 07 (2003) 003 hep-ph/0306157.

[42] B. Moussallam, Chiral sum rules for $\mathcal{L}_{(6)}^{W Z}$ parameters in its application to $\pi^{0}, \eta, \eta^{\prime}$ decays, Phys. Rev. D 51 (1995) 4939 hep-ph/9407402.

[43] M. Knecht and A. Nyffeler, Resonance estimates of $O\left(p^{6}\right)$ low-energy constants and $Q C D$ short-distance constraints, Eur. Phys. J. C 21 (2001) 659 hep-ph/0106034.

[44] Particle Data Group collaboration, K. Hagiwara et al., Review of particle physics, Phys. Rev. D 66 (2002) 010001.

[45] J. Prades, Massive spin 1 field chiral lagrangian from an extended Nambu-Jona-Lasinio model of QCD, Z. Physik C 63 (1994) 491 [hep-ph/9302246], erratum ibid. C 11 (1999) 571. 
[46] J. Bijnens, E. Gamiz, E. Lipartia and J. Prades, QCD short-distance constraints and hadronic approximations, J. High Energy Phys. 04 (2003) 055 hep-ph/0304222.

[47] K. Kawarabayashi and M. Suzuki, Partially conserved axial vector current and the decays of vector mesons, Phys. Rev. Lett. 16 (1966) 255;

Riazuddin and Fayyazuddin, Algebra of current components and decay widths of rho and $K^{*}$ mesons, Phys. Rev. 147 (1966) 1071.

[48] K. Maltman and D. Kotchan, Chiral log corrections to pseudoscalar electromagnetic selfenergies and the reliability of Dashen's theorem, Mod. Phys. Lett. A 5 (1990) 2457. G.J. Stephenson, K. Maltman and T. Goldman, QCD corrections to QED and isospin breaking in the baryon spectrum and vector meson mixing, Phys. Rev. D 43 (1991) 860. J.F. Donoghue, B.R. Holstein and D. Wyler, Electromagnetic selfenergies of pseudoscalar mesons and Dashen's theorem, Phys. Rev. D 47 (1993) 2089;

J. Bijnens, Violations of Dashen's theorem, Phys. Lett. B 306 (1993) 343 hep-ph/9302217;

D.-N. Gao, M.-L. Yan and B.-A. Li, Electromagnetic mass splittings of $\pi, a_{1}, K, K_{1}(1400)$ and $K^{*}(892)$, Phys. Rev. D 56 (1997) 4115 hep-ph/9611297.

[49] J.F. Donoghue and A.F. Perez, The electromagnetic mass differences of pions and kaons, Phys. Rev. D 55 (1997) 7075 hep-ph/9611331.

[50] J. Bijnens, G. Colangelo and G. Ecker, The mesonic chiral lagrangian of order $p^{6}$, J. High Energy Phys. 02 (1999) 020 hep-ph/9902437.

[51] D. Toublan, Lowest tensor-meson resonances contributions to the chiral perturbation theory low energy coupling constants, Phys. Rev. D 53 (1996) 6602 hep-ph/9509217, erratum ibid. D 57 (1998) 4495.

[52] T. Hambye, S. Peris and E. de Rafael, $\Delta I=1 / 2$ and $\epsilon^{\prime} / \epsilon$ in large- $N_{c} Q C D$, J. High Energy Phys. 05 (2003) 027 hep-ph/0305104.

[53] N. Isgur, C. Morningstar and C. Reader, The A1 in tau decay, Phys. Rev. D 39 (1989) 1357. 